\title{
In vivo $\mathrm{N}$-Terminomics Highlights Novel Functions of ADAMTS2 and ADAMTS14 in Skin Collagen Matrix Building
}

\author{
Cédric Leduc ${ }^{1}$, Laura Dupont ${ }^{1}$, Loïc Joannes ${ }^{1}$, Christine Monseur ${ }^{1}$, Dominique Baiwir ${ }^{2}$, \\ Gabriel Mazzucchelli ${ }^{2}$, Christophe Deroanne ${ }^{1}$, Alain Colige ${ }^{1 \dagger}$ and Mourad Bekhouche ${ }^{1,3,4 * t}$ \\ ${ }^{1}$ Laboratory of Connective Tissues Biology, GIGA-Cancer, University of Liège, Liège, Belgium, ${ }^{2}$ GIGA Proteomic Facility, \\ G/GA-Interdisciplinary Cluster for Applied Genoproteomics, University of Liège, Liège, Belgium, ${ }^{3}$ Tissue Biology and Therapeutic \\ Engineering, Centre National de la Recherche Scientifique/University of Lyon Unité Mixte de Recherche 5305, Lyon, France, \\ ${ }^{4}$ Faculté d'Odontologie de Lyon, Université de Lyon, Université Lyon 1, Lyon, France
}

\section{OPEN ACCESS}

Edited by:

Kazuhiro Yamamoto,

University of Liverpool,

United Kingdom

Reviewed by:

Fransiska Malfait,

Ghent University, Belgium

Paripok Phitsuwan,

King Mongkut's University of

Technology Thonburi, Thailand

*Correspondence:

Mourad Bekhouche

mourad.bekhouche@univ-lyon1.fr

${ }^{\dagger}$ These authors share last authorship

Specialty section:

This article was submitted to

Protein Chemistry and Enzymology,

a section of the journal

Frontiers in Molecular Biosciences

Received: 17 December 2020

Accepted: 08 February 2021

Published: 19 March 2021

Citation:

Leduc C, Dupont L, Joannes L, Monseur C, Baiwir D, Mazzucchelli G,

Deroanne C, Colige A and Bekhouche M (2021) In vivo N-

Terminomics Highlights Novel

Functions of ADAMTS2 and

ADAMTS14 in Skin Collagen

Matrix Building.

Front. Mol. Biosci. 8:643178.

doi: 10.3389/fmolb.2021.643178
A disintegrin and metalloproteinase with thrombospondin type I motif (ADAMTS)2 and ADAMTS14 were originally known for their ability to cleave the aminopropeptides of fibrillar collagens. Previous work using N-terminomic approach (N-TAILS) in vitro led to the identification of new substrates, including some molecules involved in TGF- $\beta$ signaling. Here, N-TAlLS was used to investigate the substrates of these two enzymes in vivo, by comparing the $\mathrm{N}$-terminomes of the skin of wild type mice, mice deficient in ADAMTS2, in ADAMTS14 and in both ADAMTS2 and ADAMTS14. This study identified 68 potential extracellular and cell surface proteins, with the majority of them being cleaved by both enzymes. These analyses comfort their role in collagen matrix organization and suggest their implication in inflammatory processes. Regarding fibrillar collagen, this study demonstrates that both ADAMTS2 and ADAMTS14 are involved in the processing of the aminopropeptide of alpha1 and alpha2 type $V$ collagen. It also revealed the existence of several cleavage sites in the Col1 domain and in the C-propeptide of type I collagens. In addition to collagens and other extracellular proteins, two major components of the cell cytoskeleton, actin and vimentin, were also identified as potential substrates. The latter data were confirmed in vitro using purified enzymes and could potentially indicate other functions for ADAMTS2 and 14. This original investigation of mouse skin degradomes by $\mathrm{N}$-terminomic highlights the essential role of ADAMTS2 and ADAMTS14 in collagen matrix synthesis and turnover, and gives clues to better understand their functions in skin pathophysiology. Data are available via ProteomeXchange with identifier PXD022179.

Keywords: ADAMTS, collagen, Ehlers-Danlos Syndrome (EDS), degradomics, TAILS, N-Terminomics

\section{INTRODUCTION}

Ehlers-Danlos syndrome (EDS) encompasses a group of inherited diseases caused by mutations affecting genes involved in the homeostasis of connective tissues (Beighton et al., 1998). A significant number of these genes are directly related to the biology of fibrillar collagens. Mutations in type $\mathrm{V}$ and type III procollagens are the main causes of, respectively, the "classical" and the "vascular" types of EDS. Missense mutations in type I procollagens can also lead to rare forms of classical and vascular 
EDS, while complete or partial skipping of exon six in alpha1 type I (COL1A1) or alpha2 type I (COL1A2) is responsible for arthrochalasia EDS, a condition mainly characterized by congenital bilateral hip dislocation, severe generalized joint hypermobility with multiple dislocations/subluxations and skin hyperextensibility (De Paepe and Malfait, 2012; Malfait and De Paepe, 2014). Electron microscopy of skin specimens shows loosely and randomly organized collagen fibrils with a smaller and variable diameter, and an irregular outline (Colige et al., 2004). The molecular basis behind this clinical picture is the absence of aminopropeptide cleavage of the mutated chain (either alpha1 or alpha2) since exon six encodes the cleavage site cleaved by aminoprocollagen peptidases (ADAMTS2, 3 and 14) (Colige et al., 1999). Null mutations in ADAMTS2, the main aminoprocollagen peptidase, lead also to EDS, but, surprisingly, not to the arthrochalasia EDS as reported for its mutated substrates. Instead, absence of ADAMTS2 activity causes dermatosparaxis EDS presenting with extreme skin fragility, characteristic craniofacial features, redundant skin with excessive skin folds at the wrists and ankles, umbilical hernia and severe bruising with a risk of subcutaneous hematomas and hemorrhages (Colige et al., 1999). In these patients and in animal models of deficiency in Adamts2 $\left(\mathrm{TS}^{-/-}\right.$) electron microscopy shows collagen fibrils with a highly typical hieroglyphic or ribbon-like pattern. These differences in the morphology of collagen fib rils in arthrochalasia and dermatosparaxis, while persistence of $\mathrm{N}$-propeptides is observed in both diseases, suggested that ADAMTS2 has possibly substrates other than type I procollagens.

Because of their close similarities with ADAMTS2, the consequences of mutations affecting ADAMTS3 and ADAMTS14 have been evaluated in mouse models (Janssen et al., 2016; Dupont et al., 2018). ADAMTS3 has been shown to be required for the formation of lymphatic network by its capacity to cleave pro-VEGF-C into fully active VEGF-C able to bind its receptors (Janssen et al., 2016). Regarding ADAMTS14, $\mathrm{KO}$-mice do not display obvious specific phenotype despite the fact that biallelic null mutations have been predicted to be pathogenic using dedicated browser such as "GnomAD". In the same line, mice deficient for both Adamts2 and Adamts14 $\left(\mathrm{TS}^{-/-} \mathrm{TS}^{-14^{-/}}\right.$) display an aggravated phenotype as compared to $\mathrm{TS}^{-/-}$mice, again indicating further roles and substrates for ADAMTS14 (Dupont et al., 2018). In order to have first indications about the diversity of the substrates of ADAMTS2, 3 and 14, a proteomic analysis dedicated to the identification of protease substrates (N-TAILS) was performed in cell culture models (Bekhouche et al., 2016). This study showed that the repertoire of substrates of these "so-called" aminoprocollagen peptidases extends beyond fibrillary procollagens. Although most useful because of their relatively low complexity, these in vitro models do not recapitulate the in vivo situation. As an example, fibrillary procollagens are secreted in the culture medium as uncleaved precursors, whereas in connective tissues in vivo they stay concentrated close to the producing cells and are fully matured.

In order to clarify why arthrochalasia and dermatosparaxis EDS display different clinical phenotypes (Van Damme et al.,
2016) and to investigate the diverse functions of ADAMTS2 and ADAMTS14 in vivo (Dupont et al., 2018), N-Tails analysis has been performed on skin samples from wild-type mice $(\mathrm{Wt})$ and $\mathrm{TS}_{2}^{-l-}$, $\mathrm{TS}_{1} 4^{-/-}$and $\mathrm{TS} 2^{-/-} \mathrm{TS} 14^{-/-}$.

\section{MATERIALS AND METHODS}

\section{Reagents}

The hyperbranched polyglycerol-aldehyde polymer was purchased from Flintbox (University of British Columbia, Vancouver, BC, Canada). Isobaric tag for relative and absolute quantitation (iTRAQ) labels are from AB Sciex (Concord, ON, Canada). Porcine trypsin was purchased from Promega (\#V511A; Madison, WI, United States). Ultrafiltration devices were purchased from Merck Millipore. All other reagents were purchased from Sigma-Aldrich (Saint-Quentin Fallavier, France).

\section{Cell Lines}

Fibroblasts were obtained from the dermis of healthy donors (normal fibroblasts, NF) and of a patient suffering from the dermatosparactic type of the Ehlers-Danlos syndrome (dermatosparactic fibroblasts, DF) (Nusgens et al., 1992). Cells were cultured in DMEM (Lonza) supplemented with $10 \%$ fetal bovine serum (FBS, Lonza). For collagen production, cells were cultured in DMEM supplemented with $1 \%$ FBS and $50 \mu \mathrm{g} / \mathrm{ml} \mathrm{2-}$ phospho-L-ascorbic acid (Sigma Cat\#49752). The conditioned medium was collected after $48 \mathrm{~h}$, centrifuged for $15 \mathrm{~min}$ at $4,000 \mathrm{rpm}$ at $4^{\circ} \mathrm{C}$. The supernatant was conserved at $-80^{\circ} \mathrm{C}$ before collagen purification.

\section{Skin Proteome Preparation}

The experiment was conducted in triplicate using twelve 8-weekold adult mice ( 2 males and one female of each genotype $(\mathrm{Wt}$, $\mathrm{TS}^{-1-}, \mathrm{TS}_{1} 4^{-/-}$and $\left.\left.\mathrm{TS}^{-/-} / \mathrm{TS}_{14^{-l-}}\right)\right)$. The investigation on mice was reviewed and approved by Ethics Committee for Animal Use and Care of the University of Liege (Belgium) (protocol $\mathrm{N}^{\circ} 1,109$ ). A square $\left(1 \mathrm{~cm}^{2}\right)$ of shaved skin was collected from the anteroventral part of the mice and incubated $1 \mathrm{~h}$ at $4^{\circ} \mathrm{C}$ under shaking in $3 \mathrm{ml}$ of $50 \mathrm{mM}$ HEPES sodium salt ( $\mathrm{pH} 7.5$ ), $2 \mathrm{mM}$ $\mathrm{CaCl}_{2}$ and $150 \mathrm{mM} \mathrm{NaCl}$ containing a cocktail of inhibitors of proteases and phosphatases (MS-SAFE, Sigma). Skin pieces were harvested and crushed with ceramic beads (MagNA Lyser Green Beads, Roche, n03358,941,001) using the MagNA Lyser Instrument (Roche) in $90 \%(\mathrm{~V} / \mathrm{V})$ tissue protein extraction reagent (Thermo Scientific \#78510), 10\% (V/V) HEPES sodium salt ( $\mathrm{pH} 7.5$ ), $2 \mathrm{mM} \mathrm{CaCl}, 150 \mathrm{mM} \mathrm{NaCl}$ containing the MS-SAFE inhibitors cocktail. Crushed samples were centrifuged $5 \mathrm{~min}$ at $8,000 \mathrm{rpm}$ at $4^{\circ} \mathrm{C}$ and the protein concentrations estimated (Bradford assay (Bradford, 1976)) in the collected supernatants and adjusted to $1.0 \mathrm{mg} / \mathrm{ml}$. Samples of $500 \mu \mathrm{g}$ of proteins for each condition were used for iTRAQTAILS labeling (Kleifeld et al., 2011; Bekhouche et al., 2016). Proteomic analyses were performed at the GIGA Proteomic platform on the ESI-Q Exactive (ThermoFisher) coupled with a 2D-RP/RP liquid chromatography (2D-RP/RP NanoAcquity 
UPLC, Waters, Milford, United States) for the peptide fractionation in three fractions.

\section{Proteomic Data Analysis}

Proteomic data analysis was previously described (Bekhouche et al., 2016). Briefly, in a first step, peptides were identified using Mascot (version 2.2.06; Matrix Science Inc., Boston, MA, United States) and allowing non-tryptic cleavages and two missed cleavages/peptide. Carbamidomethyl cystein was set as a fixed modification, and other modifications were set as variable: N-terminal acetyl, deamidation (NQ), Pyro-glu (N-term E), Pyro-Gln (N-term Q), Oxidation (M), iTRAQ (K), iTRAQ (Y) and iTRAQ (N-term). Peptide tolerance was set at $0.02 \mathrm{Da}$.

The tandem mass spectrometry (MS/MS) data were analyzed using the TransProteomicPipeline (TPP). The PeptideProphet and ProteinProphet software programs, embedded into TPP, were used to validate protein and peptide assignment. The nontryptic model was omitted in the PeptideProphet parameters. The error rate to validate proteins or peptides was respectively set at 2 and 5\%. Then, Clipper software was used to determine the upper and lower cutoffs corresponding to 3-sigma calculated from the normal distribution of the $\log 2$ ( $\mathrm{P}: \mathrm{C}$ ratio) from natural mature $\mathrm{N}$ termini. A Gaussian error function was used to calculate a $p$ value that reflects the probability of a peptide to be a false-positive. A peptide with a $\mathrm{P}: \mathrm{C}$ ratio above or below the 3-sigma cutoff has $99,8 \%$ chance to be dependent of the studied protease (auf dem Keller and M Overall, 2012). The cutoffs for each experiment are reported in Supplementary Table S4. The mass spectrometry proteomics data have been deposited to the ProteomeXchange Consortium via the PRIDE (PerezRiverol et al., 2019) partner repository with the dataset identifier PXD022179 and 10.6019/PXD022179.

\section{Biological Process Analysis}

The biological processes have been investigated using the Panther database (PANTHER version 14: more genomes, a new PANTHER GO-slim and improvements in enrichment analysis tools (Mi et al., 2019)). Statistical overrepresentation tests were performed using the whole Mus musculus genome as reference dataset. The $p$-Values are determined using the Fisher's Exact test corrected by the determination of the false discovery rate (fdr). The fdr was below 0.05 for all the reported biological process.

The Venn diagram were drawn using the BioVenn software (BioVenn-a web application for the comparison and visualization of biological lists using area-proportional Venn diagrams (Hulsen et al., 2008)).

\section{Purification of Recombinant ADAMTS2 and 14}

Recombinant ADAMTS2, its inactive mutant, or ADAMTS14 were produced in HEK293 cells, purified and quantified as previously described (Colige et al., 2005). Briefly, recombinant proteases were purified, using Concanavalin A-Sepharose and Heparin-Sepharose columns, from $1 \mathrm{~L}$ of serum-free medium conditioned during $48 \mathrm{~h}$. Proteases were recovered in $50 \mathrm{mM}$ Tris, $\mathrm{pH} 7.5,1 \mathrm{M} \mathrm{NaCl}, 2 \mathrm{mM} \mathrm{CaCl}$.

\section{Analysis of Fibrillar Collagens Degradation}

Collagen from dermatosparactic or normal calf skin was extracted and purified according to a reported procedure (Nusgens and Lapiere, 1979; Colige et al., 1995). Collagen from fibroblasts cultures, was concentrated by adding ethanol to the conditioned medium (see above, 2.2) at a final concentration of $33 \%(\mathrm{~V} / \mathrm{V})$. After overnight incubation at $4^{\circ} \mathrm{C}$, precipitated collagen was recovered by centrifugation $(7,000 \mathrm{rpm}, 40 \mathrm{~min}$, $\left.4^{\circ} \mathrm{C}\right)$. Pellets were solubilized in $0.1 \mathrm{M}$ acetic acid ( $\mathrm{pH} 2.9$ ) under shaking at $4^{\circ} \mathrm{C}$ for $18 \mathrm{~h}$. Non-solubilized contaminants were excluded by centrifugation $\left(17,000 \mathrm{rpm}, 40 \mathrm{~min}, 4^{\circ} \mathrm{C}\right)$ and the supernatant containing the collagen was neutralized by adding $1 \mathrm{M}$ Tris base solution (at 1/10 of the final volume). Collagen cleavage was analyzed by incubation of $10 \mu \mathrm{g}$ of type I collagen with recombinant ADAMTS2 or $14(200 \mathrm{nM}$ for $16 \mathrm{~h}$ at

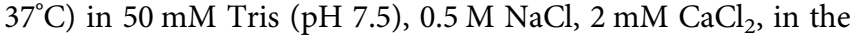
presence or absence of $25 \mathrm{mM}$ EDTA used as inhibitor of ADAMTS metalloproteinase activity. Collagens were used in native form or after thermal denaturation at $95^{\circ} \mathrm{C}$ for $10 \mathrm{~min}$. Digestion products were separated by SDS-PAGE and stained with Coomassie Blue.

\section{Analysis of Cleavage of Intracellular Substrates}

Cell lysates from human normal fibroblasts (NF) were prepared by sonication and incubated with recombinant human ADAMTS2 and/or $14\left(200 \mathrm{nM}\right.$ for $16 \mathrm{~h}$ at $\left.37^{\circ} \mathrm{C}\right)$ in $50 \mathrm{mM}$ Tris ( $\mathrm{pH} 7.5$ ), $0.5 \mathrm{M} \mathrm{NaCl}, 2 \mathrm{mM} \mathrm{CaCl}_{2}$, in the presence or absence of $25 \mathrm{mM}$ EDTA. Actin and vimentin cleavages were analyzed by Western blot using polyclonal anti-alpha-actin-1 (A2066, Sigma) and polyclonal anti-vimentin (VI008-01, Quartett).

\section{Determination of Cleavage Site Specificity}

Amino acid sequence logos, corrected by the natural abundance of amino acids in the human proteome, were generated using the iceLogo software package (Colaert et al., 2009). Analyses were based on the cleavage sites determined by proteomics for all the potential extracellular substrates, without type I collagen cleavage sites or without all fibrillar collagens.

\section{RESULTS}

\section{N-Terminomic Analysis of ADAMTS2 and ADAMTS14 Skin Degradome}

ADAMTS2 and ADAMTS14 are clearly implicated in several physio-pathological processes (Colige et al., 1999; Kesteloot et al., 2007; Dubail et al., 2010; Dupont et al., 2018; Wang et al., 2020). To better understand their in vivo roles in skin physiology and extracellular matrix homeostasis, $\mathrm{N}$-terminomic experiments have been performed on skin of mice, either wild type $(\mathrm{Wt})$,

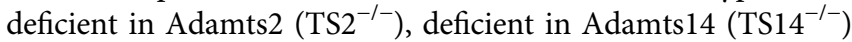



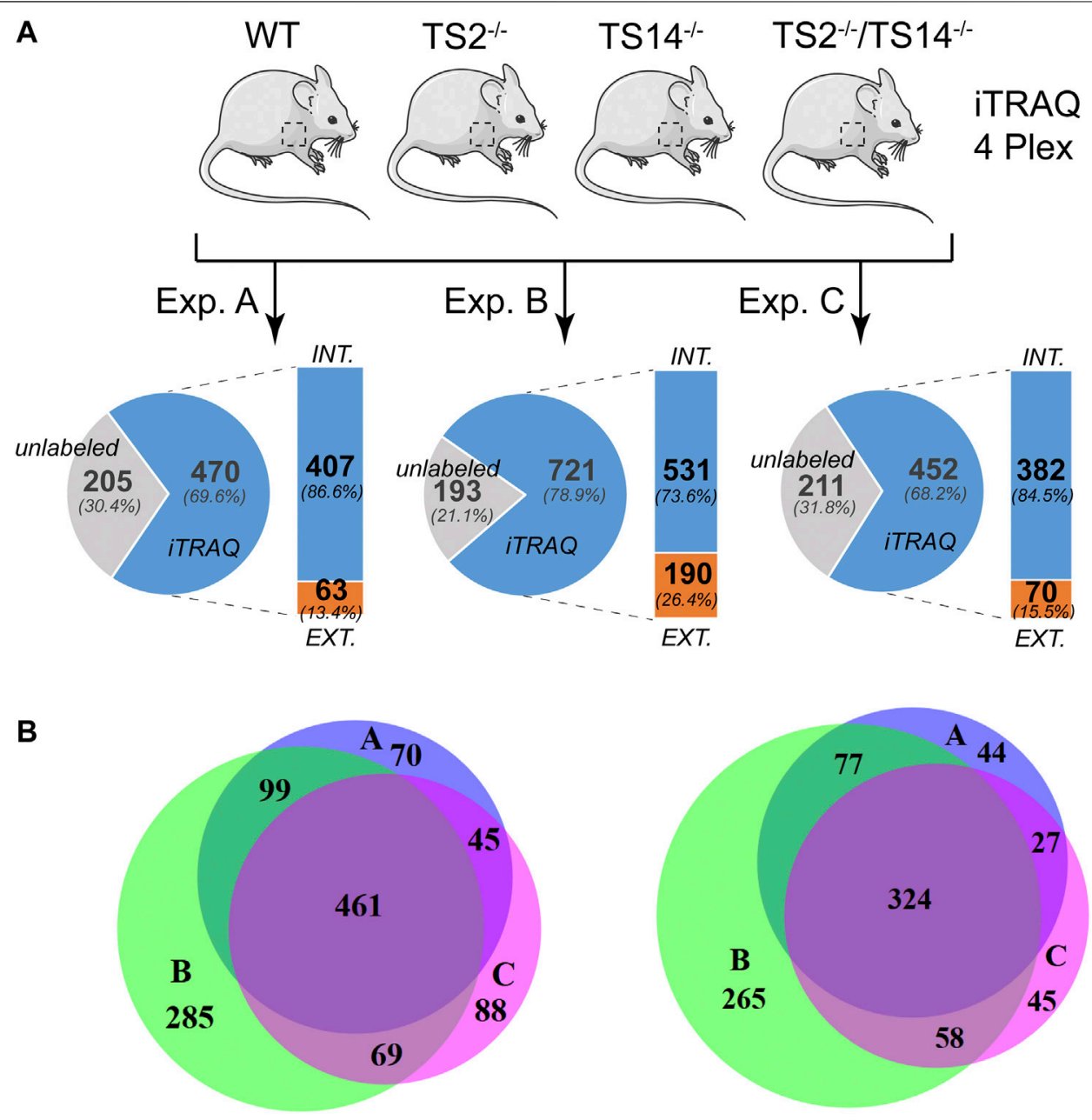

1117 total proteins

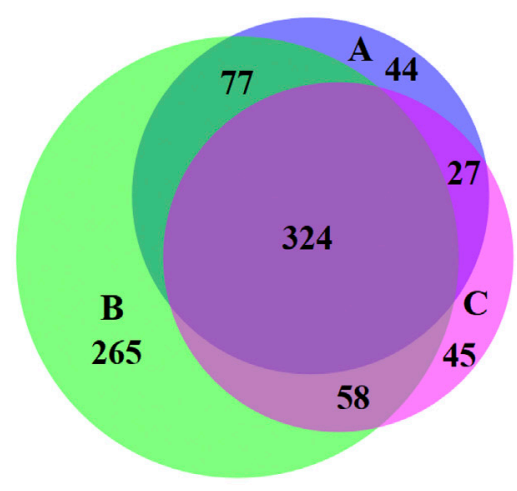

840 labeled proteins

FIGURE 1 | N-terminomic analysis of ADAMTS2 and 14 in mouse skin. (A) Schematic view of the experimental method to assess the potential substrates of ADAMTS2 and/or ADAMTS14 in mouse skin and to investigate their biological function in vivo. Three independent iTRAQ-TAlLS analyses using skin proteome of each genotypes (4-plex) have been performed. As a general procedure, a protease/control (P/C) ratio above or below the three sigma cutoff from the normal distribution of natural $\mathrm{N}$-termini is considered to be related to the studied protease. Here, the ratio $\mathrm{Wt} / \mathrm{TS} 2^{-/-}$and $\mathrm{TS}_{14^{-/-}} / \mathrm{TS}^{-/-} \mathrm{TS}_{14^{-/-}}$were used to investigate the ADAMTS2 substrates, the ratios $\mathrm{Wt} / \mathrm{TS} 14^{-/-}$and $\mathrm{TS}^{-/-} / \mathrm{TS}^{-/-} \mathrm{TS} 14^{-/-}$were used to investigate the ADAMTS14 substrates and the ratio Wt/TS2 ${ }^{-/-} \mathrm{TS}^{-4^{-/-}}$was used to investigate the substrates common to ADAMTS2 and ADAMTS14. (B) Venn diagram showing the number of specific or common total (left) or labeled (right) proteins identified in each experiment. Experiment A is in blue, B in green and C in purple. Venn diagrams were generated using the online BioVenn software (Hulsen et al., 2008).

and deficient in both Adamts2 and Adamts14 $\left(\mathrm{TS}^{-/-} \mathrm{TS}_{14^{-/-}}\right.$) (Figure 1A). For each experiment, proteins extracted from the four genotypes were labeled with specific iTRAQ labels for quantification by mass spectrometry (MS/MS). This 4-plex experiment was performed in triplicate. The proteomic analysis shows that between 68 and $79 \%$ of the proteins were labeled and have therefore been used for relative quantification. Among the labeled proteins between 13 and $26 \%$ are extracellular. By using Venn diagrams as analytical tool, a total of 1,117 proteins, including 840 with an iTRAQ labeled peptide, were identified in the three experiments, but only 41 and $38 \%$ of them were common to the three experiments, for unlabeled and labeled proteins, respectively (Figure 1B). As actual substrates might have been missed during the proteomic analyses, especially when their abundance is low, the data of these three experiments can be considered as complementary. In order to take this limitation into account but to generate also high confidence data, a labeled peptide was considered to identify a candidate substrate when it was observed in at least two experiments with a similar Protease/Control (P/C) fold change (either increased or decreased).

The degradomes (proteolytic events related to a proteolytic enzyme) are determined from the Protease/Control (P/C) ratios significantly different from the normal distribution of the natural $\mathrm{N}$-termini. They include the proteins cleaved directly by the studied protease and also indirectly by the activation of other proteases or regulatory pathways. In this study, any N-terminally iTRAQ labeled peptide was considered to be part of the 


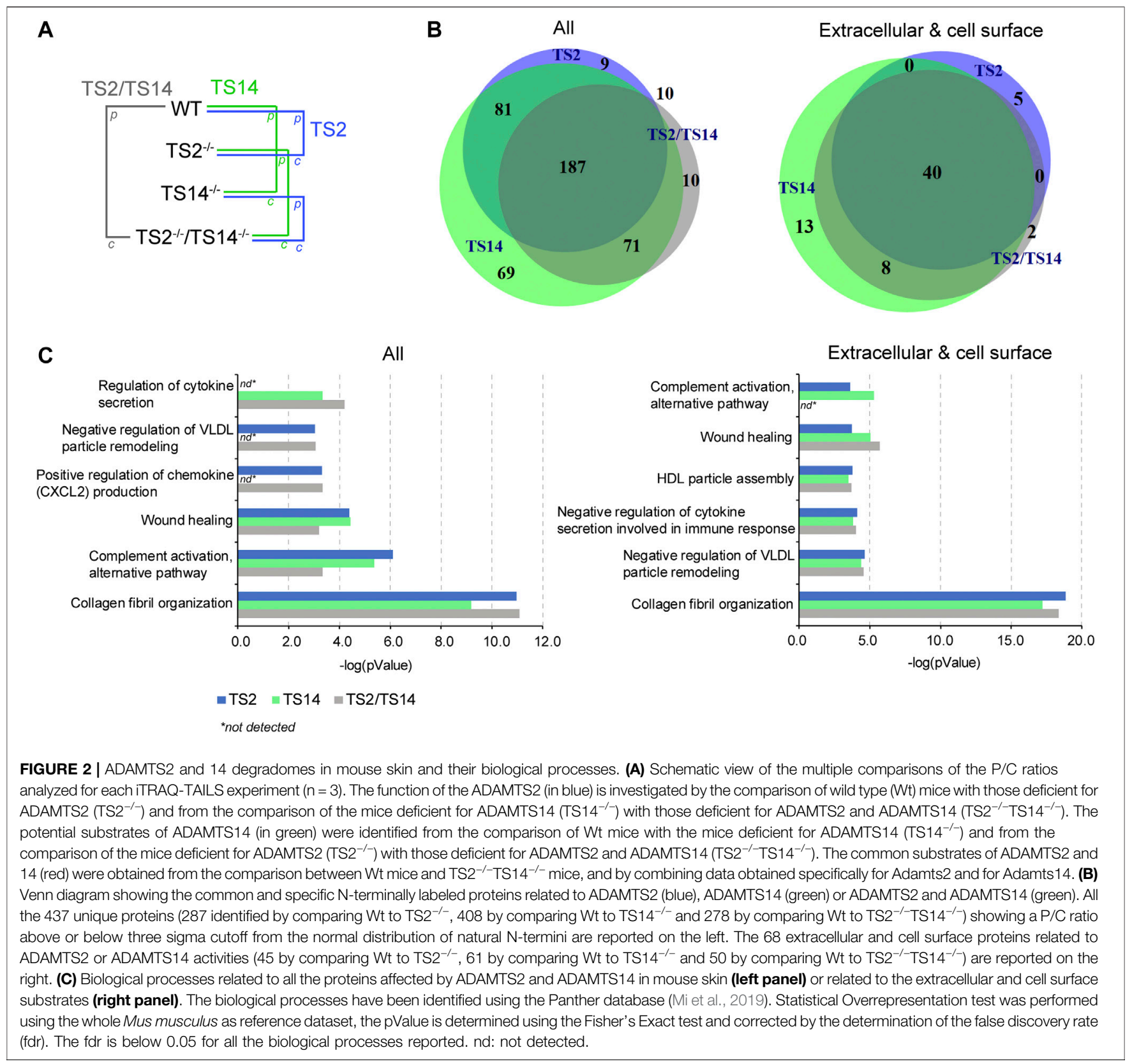

ADAMTS2 and/or ADAMTS14 degradomes when its P/C ratio was above or below a 3-sigma cut-off from the normal distribution of natural N-termini, including those obtained after the removal of the signal peptide. The degradome of ADAMTS2 has been determined from $\mathrm{Wt}$ vs $\mathrm{TS}^{-l-}$ and from $\mathrm{TS}_{14}{ }^{-/-}$vs $\mathrm{TS}^{-l-} \mathrm{TS}_{14} 4^{-1-}$ ratios, and that of ADAMTS14 has been determined from $\mathrm{Wt}$ vs $\mathrm{TS}_{1} 4^{-/-}$and from $\mathrm{TS}^{-/-}$vs $\mathrm{TS}^{-/-} \mathrm{TS} 14^{-/-}$ratios, while that of ADAMTS2 and ADAMTS14 together has been determined from $\mathrm{Wt}$ vs $\mathrm{TS}^{-1-} \mathrm{TS}_{14}{ }^{-1-}$ ratios (Figure $2 \mathrm{~A}$ ). In this analysis, the whole degradome of ADAMTS2 and/or ADAMTS14 was found to be composed of 437 proteins, including 68 proteins secreted or anchored at the cell surface. Forty extracellular and cell surface proteins were related to both ADAMTS2 and ADAMTS14 activities, while five are specific of ADAMTS2 and $21(13+8)$ of ADAMTS14. Of note, two proteins were detected only when comparing $\mathrm{Wt}$ and $\mathrm{TS}^{-1-} \mathrm{TS}_{1} 4^{-1-}$, the coagulation factor XIIIa and the mast cell protease 4 (Figure 2B). The degradomes of ADAMTS2 and ADAMTS14 are common at $59 \%(40 / 68)$ when considering specifically the extracellular and cell surface proteins, while only a 40\% (148/369) overlap is found when considering all the other proteins, showing an enrichment for common extracellular substrates (Figure 2B). Investigation of the biological processes (pantherdb.org) related to these degradomes clearly illustrated a fundamental role for these two enzymes in collagen fibril organization, which was 
TABLE 1 | Potential extracellular substrates specific or common of ADAMTS2 and/or ADAMTS14.

\begin{tabular}{|c|c|c|c|c|c|c|c|}
\hline Name & TS2 & TS14 & TS2TS14 & Name & TS2 & TS14 & TS2TS14 \\
\hline Inter alpha-trypsin inhibitor & $1.2 \pm 0.7$ & $1.9 \pm 0.7$ & $1.6 \pm 0.5$ & Galectin-1 & $1.3 \pm 0.3$ & $1.9 \pm 0.4$ & $2.1 \pm 0.4$ \\
\hline AE-binding protein 1 & $0.7 \pm 0.2$ & $2.0 \pm 0.5$ & $1.2 \pm 0.3$ & Galectin-7 & $2.0 \pm 0.6$ & $2.8 \pm 0.8$ & $4.8 \pm 1.4$ \\
\hline Alpha-2-HS-glycoprotein & $0.7 \pm 0.3$ & $2.2 \pm 1.3$ & $0.9 \pm 0.3$ & Gc-globulin & $1.7 \pm 0.8$ & $4.1 \pm 2.0$ & $6.0 \pm 3.7$ \\
\hline Alpha-2-macroglobulin & $1.7 \pm 1.0$ & $2.0 \pm 0.6$ & $2.2 \pm 0.6$ & Gelsolin & $2.6 \pm 0.7$ & $2.4 \pm 0.9$ & $5.0 \pm 1.7$ \\
\hline Annexin-8 & $6.4 \pm 2.2$ & $5.5 \pm 1.9$ & $15.0 \pm \mathrm{nd}^{\mathrm{a}}$ & H2-Q9 & $1.9 \pm 1.3$ & $2.7 \pm 1.1$ & $2.9 \pm 1.1$ \\
\hline Apolipoprotein A-I & $2.7 \pm 1.8$ & $3.3 \pm 1.9$ & $4.5 \pm 1.8$ & Hemopexin & $1.0 \pm 0.4$ & $1.9 \pm 0.5$ & $1.5 \pm 0.5$ \\
\hline Apolipoprotein H & $1.6 \pm 0.8$ & $2.6 \pm 1.2$ & $2.3 \pm 0.8$ & Ig gamma-2B & $1.8 \pm 0.5$ & $2.3 \pm 0.8$ & $3.5 \pm 1.2$ \\
\hline Beta-globin & $2.2 \pm 0.6$ & $2.9 \pm 1.1$ & $6.7 \pm 3.3$ & Ig gamma-3 & $1.1 \pm 0.4$ & $1.9 \pm 0.4$ & $1.8 \pm 0.4$ \\
\hline Biglycan & $2.8 \pm 2.4$ & $3.0 \pm 1.6$ & $3.6 \pm 1.8$ & Ig kappa chain V-V & $1.2 \pm 0.8$ & $5.1 \pm 2.7$ & $2.9 \pm 1.1$ \\
\hline Carboxypeptidase A3 & $1.6 \pm 0.4$ & $2.1 \pm 0.5$ & $3.2 \pm 1.1$ & Kallikrein j & $2.0 \pm 0.8$ & $2.4 \pm 0.6$ & $5.1 \pm 0.6$ \\
\hline Cathepsin B heavy chain & $1.3 \pm 0.6$ & $1.9 \pm 0.6$ & $1.6 \pm 0.3$ & Kininogen-1 & $2.2 \pm 0.5$ & $2.9 \pm 0.6$ & $5.5 \pm 0.2$ \\
\hline Cathepsin $\mathrm{H}$ & $1.2 \pm 0.6$ & $2.4 \pm 0.8$ & $2.0 \pm 0.6$ & Lumican & $1.6 \pm 0.7$ & $2.6 \pm 1.3$ & $2.3 \pm 0.4$ \\
\hline Cathepsin S & $1.0 \pm 0.5$ & $2.2 \pm 0.7$ & $1.5 \pm 0.4$ & Mast cell protease 4 & $1.5 \pm 0.2$ & $1.7 \pm 0.2$ & $2.4 \pm 0.5$ \\
\hline Caveolin-1 & $2.0 \pm 0.8$ & $3.1 \pm 1.4$ & $3.1 \pm 0.0$ & MIF & $1.1 \pm 0.6$ & $2.7 \pm 1.1$ & $1.8 \pm 0.5$ \\
\hline Cavin-1 & $2.1 \pm 0.4$ & $3.5 \pm 0.9$ & $8.7 \pm 0.4$ & Myelin protein zero & $1.6 \pm 0.7$ & $2.1 \pm 1.1$ & $1.7 \pm 0.4$ \\
\hline Coagulation factor XIIla & $2.5 \pm 1.0$ & $3.8 \pm 1.5$ & $7.8 \pm n d^{a}$ & Osteoglycin & $2.3 \pm 0.7$ & $2.3 \pm 0.9$ & $4.5 \pm 1.6$ \\
\hline Collagen alpha-1(I) chain & $4.2 \pm 0.5$ & $1.7 \pm 0.2$ & $6.8 \pm 1.0$ & p24 gamma-1 & $2.1 \pm 1.1$ & $3.3 \pm 1.7$ & $1.4 \pm 1.0$ \\
\hline Collagen alpha-1(III) chain & $3.7 \pm 2.2$ & $2.8 \pm 1.3$ & $5.8 \pm 2.7$ & p35/Annexin A1 & $2.7 \pm 1.2$ & $2.7 \pm 0.9$ & $7.4 \pm 3.7$ \\
\hline Collagen alpha-1(IV) chain & $1.6 \pm 0.6$ & $1.8 \pm 0.6$ & $2.2 \pm 0.4$ & p36/Annexin A2 & $2.7 \pm 1.2$ & $2.7 \pm 0.9$ & $7.4 \pm 3.7$ \\
\hline Collagen alpha-1(V) chain & $3.9 \pm 1.7$ & $4.1 \pm 1.6$ & $9.3 \pm 0.8$ & PCPE-1 & $2.2 \pm 0.4$ & $1.9 \pm 0.4$ & $3.6 \pm 0.5$ \\
\hline Collagen alpha-1(VI) chain & $2.2 \pm 0.8$ & $1.9 \pm 0.6$ & $3.4 \pm 0.9$ & PDI A6 & $1.8 \pm 0.7$ & $2.1 \pm 0.7$ & $2.9 \pm 0.9$ \\
\hline Collagen alpha-1 (XIV) chain & $3.6 \pm 0.7$ & $2.3 \pm 0.5$ & $8.2 \pm 2.5$ & Periostin & $1.0 \pm 0.5$ & $2.2 \pm 0.8$ & $1.3 \pm 0.4$ \\
\hline Collagen alpha-2(l) chain & $3.1 \pm 0.7$ & $1.6 \pm 0.2$ & $4.6 \pm 0.6$ & Proapolipoprotein A-II & $3.5 \pm 2.5$ & $4.9 \pm 3.2$ & $11.9 \pm 7.9$ \\
\hline Collagen alpha-2(V) chain & $1.8 \pm 0.3$ & $1.7 \pm 0.3$ & $2.8 \pm 0.3$ & Prolargin & $2.9 \pm 0.9$ & $2.6 \pm 0.8$ & $5.8 \pm 1.3$ \\
\hline Collagen alpha-2(VI) chain & $2.5 \pm 0.7$ & $2.2 \pm 0.6$ & $4.1 \pm 0.0$ & Protein unc-80 homolog & $1.8 \pm 0.6$ & $2.0 \pm 0.2$ & $3.5 \pm 1.4$ \\
\hline Collagen alpha-3(VI) chain & $4.7 \pm 1.3$ & $2.1 \pm 0.6$ & $8.0 \pm 1.2$ & Serpin A1c & $1.1 \pm 0.3$ & $2.4 \pm 1.0$ & $2.1 \pm 0.5$ \\
\hline Complement C3 & $1.8 \pm 1.4$ & $4.3 \pm 3.4$ & $2.3 \pm 0.8$ & Serpin A1d & $0.8 \pm 0.5$ & $2.8 \pm 1.3$ & $1.0 \pm 0.4$ \\
\hline Complement C4-B & $0.3 \pm 0.1$ & $2.7 \pm 1.0$ & $0.6 \pm 0.2$ & Serpin B6 & $4.7 \pm 2.5$ & $3.9 \pm 2.4$ & $8.0 \pm 1.9$ \\
\hline Complement factor B & $0.9 \pm 0.2$ & $2.0 \pm 0.4$ & $1.8 \pm 0.5$ & Protein serpinb6e & $1.3 \pm 0.3$ & $2.3 \pm 0.6$ & $2.5 \pm 0.1$ \\
\hline Complement factor $\mathrm{H}$ & $1.0 \pm 0.3$ & $1.8 \pm 0.5$ & $1.7 \pm 0.6$ & Serum albumin & $3.2 \pm 1.7$ & $4.2 \pm 2.4$ & $12.6 \pm 9.7$ \\
\hline Corneodesmosin & $1.5 \pm 0.4$ & $1.7 \pm 0.4$ & $2.2 \pm 0.4$ & Siderophilin & $2.2 \pm 0.7$ & $2.8 \pm 1.1$ & $5.2 \pm 2.5$ \\
\hline Cystatin-3 & $2.4 \pm 0.6$ & $2.7 \pm 0.8$ & $7.0 \pm 3.7$ & Stromelysin-1/MMP3 & $0.4 \pm 0.1$ & $1.5 \pm 0.3$ & $0.5 \pm 0.1$ \\
\hline Dermatopontin & $2.0 \pm 0.4$ & $1.6 \pm 0.2$ & $3.1 \pm 0.6$ & Susd 4 & $2.3 \pm 0.7$ & $2.4 \pm 1.1$ & $3.9 \pm 1.1$ \\
\hline Dermokine & $1.1 \pm 0.3$ & $1.4 \pm 0.3$ & $1.4 \pm 0.2$ & Transcobalamin II & $0.8 \pm 0.5$ & $2.2 \pm 1.1$ & $0.8 \pm 0.2$ \\
\hline
\end{tabular}

${ }^{a}$ nd: not determined.

The 68 extracellular and cell surface potential substrates are reported together with the average P/C ratio from at least two experiments for ADAMTS2, ADAMTS14 or ADAMTS2 and ADAMTS14. When several peptides were identified for a protein, the ratios from the peptide giving the highest value are reported for illustration. All the peptides and ratios are reported in supplemental.

expected for ADAMTS2 but was more surprising for ADAMTS14 since collagen fibrils in $\mathrm{TS} 14^{-/-}$mice appear to be normal. This analysis also shed light on their implication in lipoprotein regulation and assembly, notably by the cleavage of the proapolipoprotein A-II and apolipoprotein A-I, and in immune response by the regulation of cytokines and chemokines secretion and complement activation, notably through the cleavage of complement proteins (C3C, C4-B) and of the macrophage migration inhibitory factor (Figure $2 \mathrm{C}$, Table 1, Supplementary Figure S1).

\section{Proteomic Analysis of Type I Collagen Processing by ADAMTS2 And/or ADAMTS14 in Mouse Skin Cleavage of the Aminopropeptide of Fibrillar Collagens}

As a positive control assessing the quality and the specificity of our technical approach, we first focused on the cleavages of the aminopropeptides of type I procollagens, the primary substrates of ADAMTS2. For Col1A1 and Col1A2, the highest P/C ratios were detected at sequences corresponding to the published cleavage sites in the NC2 domain: $S_{151 \cdot 152} \mathrm{Q}$ for Col1A1 and $\mathrm{A}_{85.86} \mathrm{Q}$ for Col1A2 (Table 2). Peptides corresponding to more upstream sequences have $\mathrm{P} / \mathrm{C}$ ratios largely below 1 , evidencing the near absence of intact $\mathrm{N}$-propeptide in $\mathrm{Wt}$ skin (Supplementary Table S2). These data clearly validate the reliability of our experimental setting.

Surprisingly, several additional potential cleavage sites were also identified in the NC2 domain (Figure 3A; Table 2), some with a $\mathrm{P} / \mathrm{C}$ ratio $>2$. Some were a few amino acids downstream the "canonical" cleavage site and could therefore result from exopeptidase activity, but several others were at longer distance suggesting that ADAMTS2 cleaves within a preferred region and not exclusively at the previously reported single cleavage site.

Regarding ADAMTS14, no clear activity could be evidenced at sites cleaved by ADAMTS2, which is in line with previous studies 
TABLE 2 | Type I collagen processing in mouse skin.

Collagen a1(I) chain

Peptide Sequence

NC2 (A150-P167)

NFAS(151).(152)

QMSYGYDEKSAGVSVPGPMGPSGPR

FASQ(152).(153)

MSYGYDEKSAGVSVPGPMGPSGPR

QMSY(155).(156)

GYDEKSAGVSVPGPMGPSGPR

SYGY(157).(158)

DEKSAGVSVPGPMGPSGPR

DEKS(161).(162)AGVSVPGPMGPSGPR

EKSA (162).(163)GVSVPGPMGPSGPR

KSAG(163).(164)VSVPGPMGPSGPR Triple helix (<l>G168

VSVP(167).(168)GPMGPSGPR

VPGP(169).(170)MGPSGPR

TGPP(332).(333)

GFPGAVGAKGEAGPQGAR

PPGF(334).(335)PGAVGAKGEAGPQGAR

PGFP(335).(336)GAVGAKGEAGPQGAR

RGFP(485).(486)GADGVAGPKGPSGER

GFPG(486).(487)ADGVAGPKGPSGER

AGAQ(608).(609)GAPGPAGPAGER

PGPI(842).(843)GNVGAPGPKGPR

PPGP(889).(890)VGKEGGKGPR

AGSP(935).(936)GTPGPQGIAGQR

PGTP(938).(939)GPQGIAGQR

KNGD(1,054).(1,055)

RGETGPAGPAGPIGPAGAR

$\operatorname{NGDR}(1,055) .(1,056)$

GETGPAGPAGPIGPAGAR

RGET $(1,058) .(1,059)$

GPAGPAGPIGPAGAR

ETGP(1,060).(1,061)AGPAGPIGPAGAR

PAGP(1,063).(1,064)AGPIGPAGAR NC1 (S1182-V1453)

GYDF $(1,187) \cdot(1,188)$

SFLPQPPQEKSQDGGR

DTTL(1,224).(1,225)KSLSQQIENIR

LKSL $(1,227) .(1,228)$ SQQIENIR

\section{TS2 \\ TS14 \\ TS2TS14}

$4.2 \pm 0.5 \quad 1.7 \pm 0.2 \quad 6.8 \pm 1.0$

$2.0 \pm 0.3 \quad 1.5 \pm 0.2 \quad 2.9 \pm 0.4$

$2.4 \pm 1.0 \quad 1.6 \pm 0.3 \quad 3.3 \pm 1.1$

$3.0 \pm 1.0 \quad 1.8 \pm 0.3 \quad 5.2 \pm 2.0$

$2.0 \pm 0.3 \quad 1.5 \pm 0.2 \quad 2.9 \pm 0.5$

$1.9 \pm 0.3 \quad 1.4 \pm 0.1 \quad 2.6 \pm 0.4$

$3.7 \pm 0.3 \quad 1.4 \pm 0.1 \quad 5.1 \pm 0.2$

$8-\mathrm{P} 1181</ \mathrm{l}>)$

$1.8 \pm 0.5 \quad 1.6 \pm 0.4 \quad 2.5 \pm 0.6$

$1.8 \pm 0.5 \quad 1.5 \pm 0.4 \quad 2.6 \pm 1.0$

$2.1 \pm 0.6 \quad 1.7 \pm 0.3 \quad 3.5 \pm 1.1$

$2.3 \pm 0.8 \quad 1.7 \pm 0.5 \quad 3.5 \pm 1.7$

$2.4 \pm 0.8 \quad 1.9 \pm 0.5 \quad 4.0 \pm 1.3$

$2.6 \pm 1.0 \quad 1.7 \pm 0.4 \quad 3.8 \pm 1.5$

$1.8 \pm 0.3 \quad 1.4 \pm 0.2 \quad 2.3 \pm 0.2$

$2.2 \pm 0.4 \quad 1.6 \pm 0.3 \quad 3.1 \pm 0.3$

$2.4 \pm 0.3 \quad 1.9 \pm 0.3 \quad 4.3 \pm 0.2$

$2.4 \pm 0.6 \quad 1.4 \pm 0.3 \quad 3.1 \pm 0.8$

$2.2 \pm 0.6 \quad 1.6 \pm 0.3 \quad 3.1 \pm 1.0$

$2.6 \pm 0.8 \quad 1.6 \pm 0.4 \quad 3.8 \pm 1.3$

$2.1 \pm 0.5 \quad 2.0 \pm 0.4 \quad 3.6 \pm 0.1$

$2.0 \pm 0.4 \quad 1.4 \pm 0.2 \quad 2.6 \pm 0.5$

$1.7 \pm 0.2 \quad 1.2 \pm 0.1 \quad 2.1 \pm 0.1$

$2.4 \pm 0.7 \quad 1.6 \pm 0.4 \quad 3.5 \pm 1.1$

$2.6 \pm 0.8 \quad 1.5 \pm 0.4 \quad 3.7 \pm 1.5$$$
4.4 \pm 0.4 \quad 9.5 \pm 3.6 \quad 24.3 \pm 7.6
$$$$
2.5 \pm 0.9 \quad 2.0 \pm 0.9 \quad 3.4 \pm 0.8
$$$$
2.3 \pm 0.6 \quad 1.7 \pm 0.5 \quad 3.2 \pm 0.8
$$

$2.3 \pm 0.6 \quad 1.7 \pm 0.5 \quad 3.2 \pm 0.8$

\section{Collagen $\alpha 2(\mathrm{I})$ chain}

Peptide Sequence
NC2 (A75-P96)

NFAA(85).(86)QYSDKGVSSGPGPMGLMGPR $\quad 3.1 \pm 0.7 \quad 1.6 \pm 0.2 \quad 4.6 \pm 0.6$

FAAQ(86).(87)YSDKGVSSGPGPMGLMGPR

$2.0 \pm 0.3 \quad 2.0 \pm 0.3 \quad 1.5 \pm 0.2$

YSDK(90).(91)GVSSGPGPMGLMGPR

$2.9 \pm 0.4 \quad 1.4 \pm 0.2 \quad 2.9 \pm 0.6$

SDKG(91).(92)VSSGPGPMGLMGPR

$1.9 \pm 0.3 \quad 1.4 \pm 0.2 \quad 2.6 \pm 0.3$

DKGV(92).(93)SSGPGPMGLMGPR

$1.9 \pm 0.2 \quad 1.5 \pm 0.1 \quad 2.8 \pm 0.1$

SSGP(96).(97)GPMGLMGPR

Triple helix (G97-G1113)

GPGP(98).(99)MGLMGPR

RGIP(336).(337)GPAGAAGATGAR

RPGP(485).(486)IGPAGPR

RGTP(600).(601)GESGAAGPSGPIGSR

$1.9 \pm 0.3 \quad 1.5 \pm 0.2 \quad 2.7 \pm 0.5$

$2.5 \pm 0.8 \quad 1.8 \pm 0.5 \quad 3.9 \pm 1.6$

$2.5 \pm 0.7 \quad 1.7 \pm 0.4 \quad 3.6 \pm 1.0$

$2.4 \pm 0.7 \quad 1.6 \pm 0.4 \quad 3.5 \pm 1.3$

$2.1 \pm 0.6 \quad 1.6 \pm 0.3 \quad 3.2 \pm 1.1$

GESG(604).(605)AAGPSGPIGSR

ESGA(605).(606)AGPSGPIGSR

VGAP (636).(637)GSAGASGPGGLPGER

SGDR(699).(700)GEAGAAGPSGPAGPR

GDRG(700).(701)EAGAAGPSGPAGPR

DRGE(701).(702)AGAAGPSGPAGPR

GEAG(703).(704)AAGPSGPAGPR

EAGA(704).(705)AGPSGPAGPR

AGAP(969).(970)GPHGSVGPAGKHGNR

RGEP(987).(988)GPAGSVGPVGAVGPR

$2.0 \pm 0.2 \quad 1.3 \pm 0.1 \quad 2.5 \pm 0.4$

$2.0 \pm 0.4 \quad 1.4 \pm 0.2 \quad 2.7 \pm 0.5$

$2.3 \pm 0.8 \quad 1.7 \pm 0.4 \quad 3.5 \pm 1.2$

$1.9 \pm 0.4 \quad 1.6 \pm 0.3 \quad 3.0 \pm 0.7$

$1.9 \pm 0.3 \quad 1.5 \pm 0.2 \quad 2.8 \pm 0.3$

$2.4 \pm 0.6 \quad 1.7 \pm 0.4 \quad 3.8 \pm 1.1$

$2.0 \pm 0.2 \quad 1.3 \pm 0.1 \quad 2.5 \pm 0.4$

$2.1 \pm 0.4 \quad 1.5 \pm 0.2 \quad 3.0 \pm 0.7$

$2.2 \pm 0.5 \quad 1.6 \pm 0.3 \quad 3.4 \pm 0.7$

$2.5 \pm 0.8 \quad 1.6 \pm 0.4 \quad 3.7 \pm 1.4$

EPGP(989).(990)AGSVGPVGAVGPR

$2.2 \pm 0.6 \quad 1.5 \pm 0.3 \quad 3.2 \pm 1.1$

PGPA(990).(991)GSVGPVGAVGPR

$1.9 \pm 0.2 \quad 1.3 \pm 0.1 \quad 2.4 \pm 0.4$

AGSV(993).(994)GPVGAVGPR

SVGP(995).(996)VGAVGPR

$\operatorname{LKGY}(1,031) \cdot(1,032)$

SGLQGLPGLAGLHGDQGAPGPVGPAGPR

$2.3 \pm 0.5 \quad 1.5 \pm 0.2 \quad 3.3 \pm 0.9$

$1.7 \pm 0.2 \quad 1.6 \pm 0.2 \quad 2.6 \pm 0.3$

$2.2 \pm 0.6 \quad 1.9 \pm 0.5 \quad 3.6 \pm 1.1$

$2.6 \pm 1.2 \quad 1.7 \pm 0.5 \quad 3.1 \pm 1.0$

QGLPGLAGLHGDQGAPGPVGPAGPR

GLPG $(1,039) .(1,040)$

LAGLHGDQGAPGPVGPAGPR

LPGL $(1,040) .(1,041)$

AGLHGDQGAPGPVGPAGPR

PGLA(1,041).(1,042)

GLHGDQGAPGPVGPAGPR

LAGL $(1,043) .(1,044) H G D Q G A P G P V G P A G P R$

GLHG $(1,045) .(1,046)$ DQGAPGPVGPAGPR

LHGD $(1,046) .(1,047)$ QGAPGPVGPAGPR

HGDQ $(1,047) .(1,048)$ GAPGPVGPAGPR

GDQG $(1,048) .(1,049)$ APGPVGPAGPR

RSGQ(1,076).(1,077)PGPVGPAGVR NC1 (Y1114-K1372)

DATL $(1,145) .(1,146)$ KSLNNQIETLLTPEGSR

LKSL $(1,148) .(1,149) N N Q I E T L L T P E G S R$

RLPF $(1,347) .(1,348)$ LDIAPLDIGGADQEFR
$2.9 \pm 0.6 \quad 1.9 \pm 0.5 \quad 5.6 \pm 2.4$

$2.3 \pm 0.4 \quad 1.3 \pm 0.2 \quad 2.8 \pm 0.3$

$2.8 \pm 1.2 \quad 2.5 \pm 1.2 \quad 6.1 \pm 2.9$

$2.0 \pm 0.3 \quad 1.6 \pm 0.2 \quad 2.9 \pm 0.5$

$1.5 \pm 0.4 \quad 1.8 \pm 0.2 \quad 2.7 \pm 0.9$

$2.2 \pm 0.3 \quad 1.5 \pm 0.2 \quad 3.2 \pm 0.1$

$1.8 \pm 0.2 \quad 1.3 \pm 0.1 \quad 2.4 \pm 0.4$

$2.0 \pm 0.2 \quad 1.4 \pm 0.2 \quad 2.7 \pm 0.5$

$2.2 \pm 0.3 \quad 1.5 \pm 0.2 \quad 3.2 \pm 0.1$

$3.9 \pm 3.4 \quad 6.0 \pm 5.6 \quad 3.2 \pm 1.0$

$1.6 \pm 0.4 \quad 2.0 \pm 0.6 \quad 2.7 \pm 0.8$

$3.0 \pm 0.7 \quad 2.0 \pm 0.6 \quad 5.3 \pm 1.2$

Cleavage sites observed by proteomics of ADAMTS2 (TS2) and ADAMTS14 (TS14) within $\alpha 1$ and $\alpha 2$ chains of type I collagen. Each cleavage site has been observed at least in two experiments. The sequences of the cleavage sites are reported in bracket according to the UniprotKB numbering. The classical N-propeptide cleavage sites are highlighted in grey.

(Dupont et al., 2018) showing that type I collagen is fully processed in the $\mathrm{TS}_{1} 4^{-/-}$mice, and further confirming the specificity of our analyses. Most interestingly, the $\mathrm{P} / \mathrm{C}$ ratios were, however, higher in $\mathrm{TS}^{-/-} \mathrm{TS}_{1} 4^{-/-}$than in $\mathrm{TS}^{-/-}$mice suggesting that, in the absence of ADAMTS2, ADAMTS14 can display some aminoprocollagen peptidase activity in vivo (Table 2). 
A
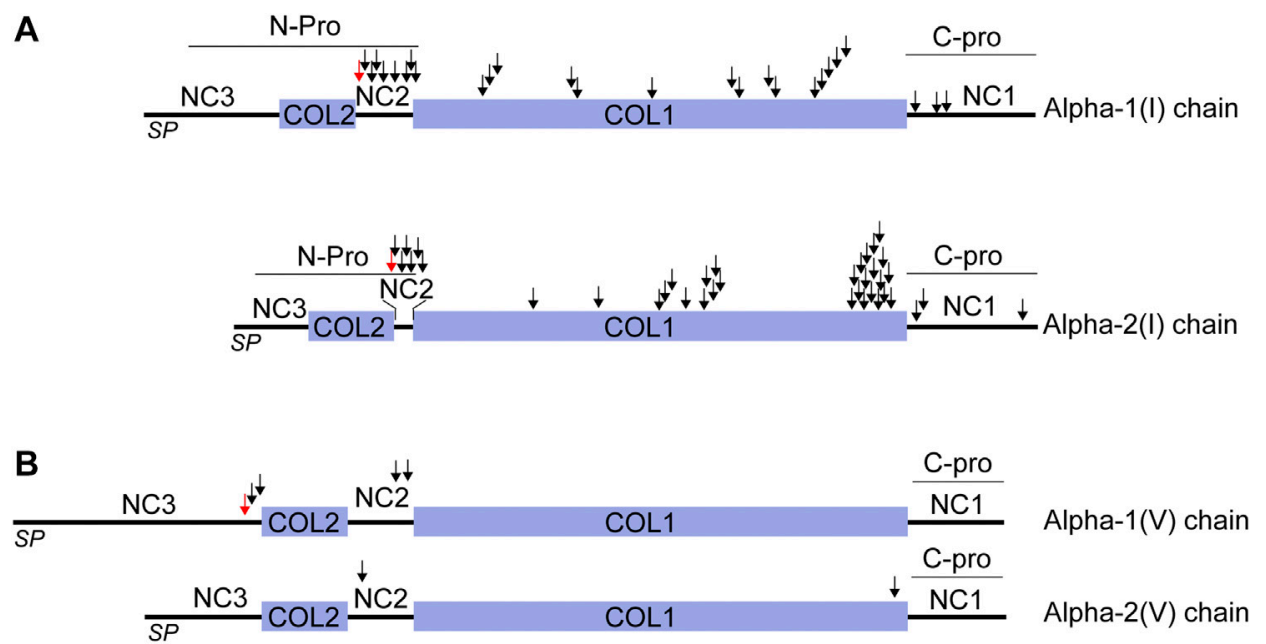

\begin{tabular}{|l|c|c|c|}
\hline & \multicolumn{4}{c}{ TS2 } & \multicolumn{1}{c|}{ TS14 } & TS2TS14 \\
\hline Aleavage sites & \multicolumn{4}{|c|}{ Alpha-1(V) chain } \\
\hline PGMP(435).(436)ANQD & $3.9 \pm 1.7$ & $4.1 \pm 1.6$ & $9.3 \pm 0.8$ \\
\hline MPAN(437).(438)QDTI & $2.5 \pm 0.6$ & $1.9 \pm 0.4$ & $3.9 \pm 0.3$ \\
\hline QDTI(441).(442)FEGI & $2.2 \pm 0.6$ & $1.8 \pm 0.5$ & $3.4 \pm 0.7$ \\
\hline AGSK(535).(536)GPMV & $1.5 \pm 0.4$ & $2.1 \pm 0.5$ & $3.0 \pm 0.8$ \\
\hline MVSA(541).(542)QESQ & $1.6 \pm 0.3$ & $1.8 \pm 0.3$ & $2.7 \pm 0.7$ \\
\hline \multicolumn{4}{|c|}{ Alpha-2(V) chain } \\
\hline SRPF(189).(190)SAQM & $1.8 \pm 0.3$ & $1.7 \pm 0.3$ & $2.8 \pm 0.3$ \\
\hline QGPP(903).(904)GATG & $2.6 \pm 0.9$ & $1.8 \pm 0.4$ & $4.3 \pm 1.6$ \\
\hline
\end{tabular}

FIGURE 3 | ADAMTS2 and/or ADAMTS14 cleavages of type I and V collagens. Schematic view of the pro alpha-1(I) and alpha-2(I) chains showing the signal peptides (PS), the main triple helix collagenous domain (COL1) and the short triple helix collagenous domain (COL2) and the N- and C-propeptides (N- and C-Pro) (A). Schematic view of the pro alpha-1(V) and alpha-2(V) chains showing the collagenous (COL) and non collagenous (NC) domains (B). The ADAMTS2 and ADAMTS14 cleavage sites are indicated by arrows. The red arrows correspond to the already reported ADAMTS2 cleavage sites (Colige et al., 2005 ; Bekhouche and Colige, 2015). Table showing the $P / C$ ratio for type $V$ collagens, according to the cleavage sites observed by proteomic. Ratios above the average $3 \sigma$ cut-offs are highlighted in green. The previously described ADAMTS2 cleavage site within alpha-1(V) chain is in bold (C).

The processing of the aminopropeptide of type $\mathrm{V}$ procollagens is still a matter of controversy, especially in vivo. Processing of Col5A1 has been reported to be performed by BMP1 (within the NC3 domain) and by ADAMTS2 (within the NC3 domain upstream of the Col2 domain) (Figure 3B). However, no cleavage has been reported in the NC2 domains of Col5A1 or Col5A2, while their sequence and localization between two collagen domains are similar to those of type I, II and III fibrillar procollagens which are all processed in this region. Several peptides N-terminally iTRAQ labeled were identified in the large $\mathrm{N}$-terminal domain of Col5A1 encompassing the Col2, NC2 and NC3 domains (Figure 3B). As opposed to what was observed for type I procollagens, the $\mathrm{P} / \mathrm{C}$ ratios were usually similar for ADAMTS2 and ADAMTS14, suggesting that both enzymes can process Col5A1 with the same efficacy (Figure 3C). Accordingly, the $\mathrm{P} / \mathrm{C}$ ratios were much higher when comparing $\mathrm{Wt}$ and $\mathrm{TS}^{-l-} \mathrm{TS}_{14}^{-l-}$ samples. The main processing site $\left(\mathrm{P}_{435 \cdot 436} \mathrm{~A}\right.$, upstream of the Col2 domain) was identical to that described previously using recombinant proteins (Colige et al., 2005). As for Col1A1 and Col1A2, other cleavage sites were also identified in this region of Col5A1, two with $\mathrm{P} / \mathrm{C}>2\left(\mathrm{~N}_{437.438} \mathrm{Q}\right.$ and $\left.\mathrm{I}_{441 \cdot 442} \mathrm{~F}\right)$ and one with a $\mathrm{P} / \mathrm{C}$ of $0.4\left(\mathrm{Q}_{438 \cdot 439} \mathrm{D}\right)$ reflecting a preferential cleavage at $\mathrm{N}_{437 \cdot 438} \mathrm{Q}$. The $\mathrm{P} / \mathrm{C}$ of the other labeled peptides was not affected by the presence or absence of ADAMTS2 and ADAMTS14, which confirms the specificity of the cleavages with a $\mathrm{P} / \mathrm{C}$ ratio $>2$.

Most interestingly, two cleavage sites were also identified in the NC2 domain of Col5A1, including one at an A. Q site as in Col1A2 (Figure 3B). Altogether these data indicate that ADAMTS2 and ADAMTS14 can process the N-terminal portion of Col5A1 at three different sites, meaning that Col5A1 is present in the skin under forms (including that generated by BMP1 cleavage) having N-terminal noncollagenous extremity of different size and bulkiness.

N-terminally iTRAQ-labeled peptides corresponding to the NC2 domain of Col5A2 were also detected. Although the P/C 
ratios were lower than for Col1A1, Col1A2 and Col1A5, it clearly indicates that the N-propeptide of Col5A2 can be processed by ADAMTS2 and ADAMTS14, but probably at a reduced rate.

Similar to our previous study in vitro, the cleavage of the $\mathrm{N}$-propeptide of Col3A1 was not seen in this experiment, probably because the corresponding peptide was not isolated and therefore not analyzed during the MS/MS step.

\section{Cleavages Outside of the Aminopropeptide}

Having confirmed and further documented the processing of the aminopropeptides of fibrillar collagens, we were also intrigued by the presence of potential cleavage sites elsewhere in type I collagen chains. In a previous work performed in vitro using human cells (Bekhouche et al., 2016), we identified several peptides corresponding to the Coll domain and the C-propeptide of Col1A1 and Col1A2 that were possibly generated by ADAMTS2 or ADAMTS14 (Supplementary Table S3). However, we hypothesized that this could be an artifact linked to the in vitro conditions, such as incomplete folding of the triple helical domains leading to an increased sensitivity to proteases. Here, dozens of peptides corresponding to the Coll domain of Col1A1 and Col1A2 were found to be N-terminally iTRAQ labeled. For both Col1A1 and Col1A2, the $\mathrm{P} / \mathrm{C}$ ratios were, on average, between 1.5 and two when considering ADAMTS2, and between 1.0 and 1.5 for ADAMTS14 (Supplementary Tables S2, S3). However, similarly to what was observed for the processing of the aminopropeptides, these ratios were significantly higher when comparing $\mathrm{Wt}$ and $\mathrm{TS}^{-/-} \mathrm{TS}^{-/-}$skin samples, suggesting that ADAMTS2 and ADAMTS14 can cleave at identical sites. Some "hot spot" regions characterized by several contiguous cleavage sites were found, as, for example, at positions 333 to 337 and 827 to 838 of Col1A1 as well as at positions 988 to 996 of Col1A2 (Table 2). They can be generated by direct cleavages operated by ADAMTS2 and 14 in more sensitive domains or, alternatively, by a single cleavage followed by progressive degradation by exopeptidases. In both cases however, it means that the observed individual $\mathrm{P} / \mathrm{C}$ ratios give an underestimation of the actual cleavage activity in the concerned region.

Finally, peptides corresponding to cleavages in the C-propeptides were also found. As opposed to our observations for Coll domain, the $\mathrm{P} / \mathrm{C}$ ratios were similar for both ADAMTS2 and ADAMTS14, again illustrating the specificity in the identification of potential cleavage sites. For CollA1, one site $\left(\mathrm{F}_{1187 \cdot 1188} \mathrm{~S}\right)$ is located upstream of the C-propeptide cleavage site by BMP1 $\left(\mathrm{A}_{1207 \cdot 1208} \mathrm{D}\right)$ while the two others are located about 20 amino acids downstream. For Col1A2, three sites were identified, all after the cleavage site for BMP1 (Table 2).

\section{Confirmation of the Cleavage of Type I Collagen Within the Col1 Domain and the C-Propeptide}

To confirm our iTRAQ data showing multiple cleavages in the Col1 triple helical domain and in the C-propeptide of type I collagens, we performed in vitro assays using recombinant enzymes and collagen purified from the skin of dermatosparactic calf which is characterized by the persistence of the aminopropeptides in about $80 \%$ of type I collagen molecules (Figure 4A, lane 1). This particular substrate was chosen because it provides the opportunity to have an internal control consisting in the processing of the aminopropeptide of CollA1 and Col1A2. This cleavage (conversion of $\mathrm{pNa} 1$ and $\mathrm{pNa} 2$ into a1 and a 2 chains) was almost complete in the presence of recombinant ADAMTS2, but much reduced in the presence of ADAMTS14 in accordance with its lower aminoprocollagen peptidase activity (Figure 4A, lanes 2 and 4, respectively). When looking at lower molecular weight products (Figures 4C,E), the released $\mathrm{pNa}$ propeptide was observed with an apparent $30 \mathrm{kDa} \mathrm{MW}$ and was mainly found in the presence of recombinant active ADAMTS2 (lanes 2 and 6). The presence of other bands and of "trails", covering the entire migration lanes and corresponding to cleavages of collagen in multiple sites, were also identified (lane 2), including in the presence of recombinant ADAMTS14 (lane 4). Since ADAMTS14 has only a reduced aminoprocollagen peptidase activity, it shows that the two types of activity are not related.

The same type I collagen preparation was also used as substrate after heat denaturation to verify whether disruption of the triple helical folding impacts the sensitivity to cleavage. Processing of the aminopropeptides by ADAMTS2 or ADAMTS14 was still observed, but marked differences were also visible as compared to gels obtained with native collagen (compare panels a, c, e to panels b, d, f of Figure 4), such as the presence of products at 97 and $110 \mathrm{kDa}$ observed in the presence of ADAMTS2 and 14 (lanes 2, 4 and 6 on each panel), and at $105 \mathrm{kDa}$ specifically in the presence of ADAMTS14 (lanes four on each panel). Additional discrete degradation products of lower MW were also identified as well as more pronounced degradation trails along the entire migration lanes (Figure 4, lanes 2, 4, 6; panels $\mathrm{d}$ and $\mathrm{f}$ ).

The presence of cleavage sites within the C-propeptide of Col1 A1 and Col1A2 was also evidenced by our iTRAQ analyses on in vitro assays. Since collagen purified from dermatosparactic skin lacks the C-propeptide, we used collagen produced by human dermatosparactic fibroblasts in culture which is mainly secreted as complete procollagen still retaining its two propeptides (pro-alpha one and pro-alpha 2). After incubation with active ADAMTS2 (Figure 5, lane 2), proa1 and proa2 are converted into pCa1 and pCa2, respectively, as a result of the aminoprocollagen peptidase activity of ADAMTS2. Fully processed alpha1 was also observed while similar amount of $\mathrm{pNa}$ was absent in the control (lane 1) suggesting that it is produced by cleavage of the $\mathrm{N}$ - and $\mathrm{C}$-propeptides of the proal chain. In line with this hypothesis, the amount of alpha2 chain recovered after incubation with ADAMTS2 exceeded the amount of $\mathrm{pNa} 2$ in the control. Moreover, the relative intensity of $\mathrm{pCa} 2$ was lower after incubation with ADAMTS2 (lane 2) than expected from the intensity of proa 2 in the control. These two observations clearly suggest some cleavage of the C-propeptide of proa2. 

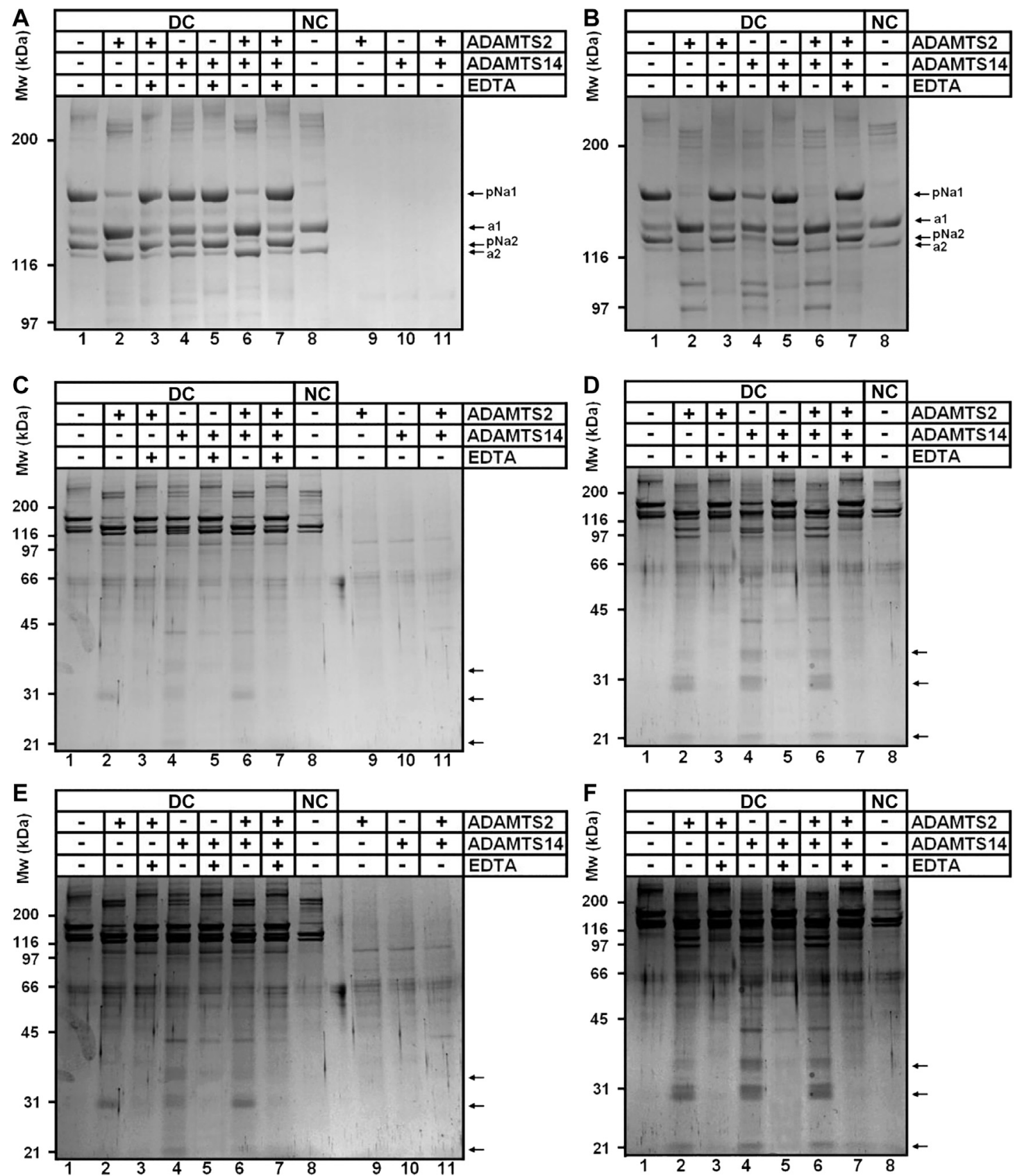

FIGURE 4 | Collagen digestion from calf skin by recombinant human ADAMTS2 and/or ADAMTS14 (A-F) Collagen from dermatospatactic calf (DC) skin has been heat denatured $\left(10 \mathrm{~min}\right.$ at $\left.95^{\circ} \mathrm{C}\right)(\mathbf{B}, \mathbf{D}, \mathbf{F})$ or not $(\mathbf{A}, \mathbf{C}, \mathbf{E})$ before addition of recombinant human TS2 and/or TS14 overnight at $37^{\circ} \mathrm{C}$. Digestion products have been analyzed by $6.25 \%$ (A, B) or $10 \%$ (C-F) SDS-PAGE stained by Coomassie blue. Pictures at (E) and (F) correspond respectively to (C) and (D) with a higher contrast to emphasize the degradation trail. Collagen from normal calf skin (NC) have been used as control for the identification of the fully processed a1 and $\alpha 2$ chains. Products at $31 \mathrm{kDa}(\mathbf{C}, \mathbf{E})$ correspond to the $\mathrm{N}$-terminal propeptide released mainly by ADAMTS2 when type I collagen is in its native form. Additional low MW products can be observed after incubation of denatured collagen with ADAMTS14 and ADAMTS2.

Regarding ADAMTS14, only a low aminoprocollagen peptidase activity was observed, as expected, as illustrated by the presence of low amounts of $\mathrm{pCa} 2$ generated from proa2 (lane 3). However, accumulation of $\mathrm{pNa} 2$ was clearly observed indicating that ADAMTS14 can cleave the C-propeptide of Col1A2 more efficiently than its $\mathrm{N}$-propeptide. 


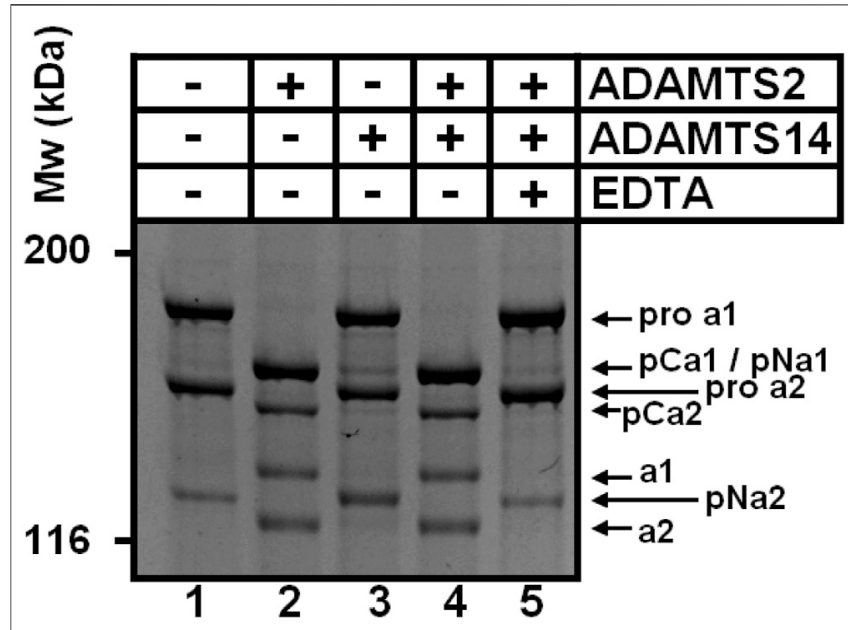

FIGURE 5 | Cleavage of type I procollagen. The collagen substrate, mainly in the form of pro- $\alpha 1 \mathrm{l}$ and pro- $\alpha 2 \mathrm{I}$, was recovered from culture medium conditioned by dermatosparactic fibroblasts, and then incubated with recombinant human ADAMTS2 and/or ADAMTS14 during $18 \mathrm{~h}$ at $37^{\circ} \mathrm{C}$. Digestion products were analyzed by $6.25 \%$ SDS-PAGE stained by Coomassie blue. The cleavage of pro- $\alpha 2$ into $\mathrm{pC} \alpha 2$ and mature $\alpha 2$ demonstrates that ADAMTS2 displays a procollagen C-proteinase activity in addition to its aminoprocollagen peptidase property.

\section{Other Potential Substrates Involved in Collagen Fibril Formation}

Several proteoglycans, non-fibrillar collagens and matricellular proteins are known to be involved in the regulation of collagen fibril formation and functions. Some of them were found to be potential substrates of ADAMTS2 and/or ADAMTS14 based on $\mathrm{P} / \mathrm{C}$ ratios significantly $>2$ (Table 1 ). They are therefore mentioned, although cleavages were not confirmed by other methods, since their processing could be involved in the clinical features found in dermatosparactic EDS and other connective tissue disorders.

Two sites of cleavage in type XIV collagen were found for both ADAMTS2 and ADAMTS14: at positions 630 (AQQY.LEID) within the fifth fibronectin type-III domain and 1,636 (MARY.TAIL) within the third collagen like domain. Identical sites for both enzymes strongly suggest that the cleavages are real and that type XIV collagen is a true substrate. Potential cleavages were also found in COL4A1 $\left(\mathrm{G}_{1438 \cdot 1439} \mathrm{~T}\right)$, at a position corresponding to the release of the arresten cryptic bioactive fragment and in type VI collagens: (1) in the first VWFA domain of COL6A1 $\left(\mathrm{F}_{186 \cdot 187} \mathrm{~S}\right)$; (2) in the VWFA1 and VWFA2 domains of COL6A2 $\left(\mathrm{F}_{116 \cdot 117} \mathrm{~S}\right.$ and $\left.\mathrm{F}_{141 \cdot 142} \mathrm{~A}\right)$ and (3) in the VWFA6 domain of COL6A3 $\left(\mathrm{F}_{1051 \cdot 1052} \mathrm{~A}\right)$. Besides collagens, potential proteolytic cleavages were also found in PCPE-1 (a co-factor stimulating the processing of the C-propeptide of fibrillar collagens by BMP-1) and in three proteoglycans regulating matrix assembly: biglycan, lumican and osteoglycin. Finally, the degradomes of ADAMTS2 and ADAMTS14 in mouse skin point out several proteins involved in the immune system such as immunoglobulins, complement proteins ( $\mathrm{C} 3, \mathrm{C} 4-\mathrm{B}$, factor $\mathrm{B}$, factor $\mathrm{H}$ ), the macrophage inhibitory factor or annexins $\mathrm{A} 8$,
A1 and A2 known to be involved in leukocyte recruitment and activation (Swisher et al., 2007; Poeter et al., 2014; Sugimoto et al., 2016).

\section{Potential Intracellular Cleavages}

This work was primarily focused on extracellular and cell surface degradome since ADAMTS2 and ADAMTS14 are secreted proteases. Unexpectedly however, numerous cleavage sites related to the presence/absence of ADAMTS2 and/or ADAMTS14 were found in intracellular proteins (Supplementary Table S1), including actins and vimentin (Figure 6A). On average, $\mathrm{P} / \mathrm{C}$ ratios for actin were higher for ADAMTS14 than for ADAMTS2, as opposed to what was seen for the N-propeptides cleavages, and even higher when comparing Wt skin and $\mathrm{TS}^{-l-} \mathrm{TS}^{-1-} 4^{--}$skins. Of note also, cleavage sites in actin-2, also known as gamma-actin, are identical to the corresponding cleavage sites in actin-1 which suggests their specificity. In order to confirm these surprising observations, fibroblasts extracts were incubated in the presence of purified ADAMTS2 and/or ADAMTS14 with or without a saturating concentration of EDTA used as inhibitor (Figure 6B). In the absence of ADAMTS and EDTA, actin was detected as a single band of $42 \mathrm{kDa}$ with an antibody raised against the C-terminal decapeptide of alpha-actin-1, demonstrating absence of cleavage by intracellular endogenous proteases, while a second product of about $40 \mathrm{kDa}$ was observed after incubation with ADAMTS2, which would correspond to cleavages at positions $\mathrm{V}_{19 \cdot 20} \mathrm{~K}$ and/or $\mathrm{A}_{21 \cdot 22} \mathrm{G}$ in alpha-actin-1 (Figure 6A). This product was also seen with ADAMTS14, in addition to a second one at about $34-35 \mathrm{kDa}$ which was not observed with ADAMTS2. Remarkably, only discrete bands were seen, with no trace of smear which would indicate multiple cleavages at specific positions.

Regarding vimentin, several peptides with $\mathrm{N}$-terminal iTRAQ labeling were detected, some with $\mathrm{P} / \mathrm{C}$ ratio slightly but significantly higher in the presence of ADAMTS2 or ADAMTS14 (Figure 6A). For confirmatory purposes Western blot analysis was performed on fibroblast extracts incubated or not with purified ADAMTS2 or ADAMTS14. In the control samples, vimentin appeared as two major bands at $48 \mathrm{kDa}$ and at $58 \mathrm{kDa}$ the latter likely corresponding to the full-length protein. Additional minor products at 53,50, 46 and $39 \mathrm{kDa}$ were also visible (Romano et al., 2020) (Figure 6C). Upon incubation with ADAMTS2 or 14, the intensity of bands at 58 and $53 \mathrm{kDa}$ were markedly reduced, accompanied by an increased intensity of the products at 48 and $39 \mathrm{kDa}$ and the presence of additional bands at 37 and $32 \mathrm{kDa}$. These data clearly suggest the existence of several cleavage sites by ADAMTS2 and ADAMTS14 but were not investigated further.

\section{Cleavage Site of ADAMTS2 and ADAMTS14 in Mouse Skin}

The cleavage sites observed by $\mathrm{N}$-terminomics were used to evaluate cleavage sites enrichment linked to the presence of ADAMTS2 and ADAMTS14. All the potential cleavage sites were used, in a first step, to establish the privileged consensus 


\begin{tabular}{|c|c|c|c|}
\hline \multicolumn{4}{|l|}{ A } \\
\hline Cleavage sites & TS2 & TS14 & TS2TS14 \\
\hline \multicolumn{4}{|c|}{ Alpha-ACTIN-1 } \\
\hline VCDN(14).(15)GSGL & $1.4 \pm 0.3$ & $2.1 \pm 0.6$ & $2.5 \pm 0.5$ \\
\hline SGLV(19).(20)KAGF & $2.1 \pm 0.4$ & $2.2 \pm 0.4$ & $4.5 \pm 1.1$ \\
\hline LVKA(21).(22)GFAG & $2.1 \pm 0.7$ & $2.2 \pm 0.8$ & $3.6 \pm 0.8$ \\
\hline PTLL(107).(108)TEAP & $2.0 \pm 1.0$ & $3.9 \pm 2.4$ & $3.2 \pm 3.1$ \\
\hline KDLY(296).(297)ANNV & $1.8 \pm 0.6$ & $2.4 \pm 0.9$ & $3.5 \pm 1.1$ \\
\hline KQEY(364).(365)DEAG & $2.4 \pm 0.7$ & $2.6 \pm 0.6$ & $5.8 \pm 1.7$ \\
\hline \multicolumn{4}{|c|}{ Gamma-ACTIN } \\
\hline KDLY(294).(295)ANTV & $1.6 \pm 1.0$ & $3.5 \pm 1.6$ & $2.7 \pm 2.0$ \\
\hline QQMW(356).(357)ISKQ & $1.8 \pm 0.6$ & $1.9 \pm 0.5$ & $2.6 \pm 0.4$ \\
\hline KQEY(362).(363)DESG & $1.8 \pm 0.3$ & $1.9 \pm 0.3$ & $3.4 \pm 0.2$ \\
\hline \multicolumn{4}{|c|}{ VIMENTIN } \\
\hline SNRS(29).(30)YVTT & $1.7 \pm 0.5$ & $1.7 \pm 0.5$ & $3.0 \pm 1.5$ \\
\hline RSLY(53).(54)SSSP & $1.9 \pm 0.5$ & $1.9 \pm 0.5$ & $3.8 \pm 0.6$ \\
\hline SVDF(86).(87)SLAD & $1.1 \pm 0.3$ & $1.9 \pm 0.5$ & $1.8 \pm 0.6$ \\
\hline DFSL(88).(89)ADAI & $1.2 \pm 0.4$ & $1.7 \pm 0.6$ & $1.3 \pm 0.1$ \\
\hline SLAD(90).(91)AINT & $1.4 \pm 0.5$ & $1.5 \pm 0.5$ & $1.6 \pm 0.4$ \\
\hline ).(132)LAEL & $1.7 \pm 0.6$ & $1.9 \pm 0.6$ & $2.4 \pm 0.8$ \\
\hline KILL(132).(133)AELE & $1.8 \pm 0.6$ & $2.3 \pm 0.5$ & $3.4 \pm 0.5$ \\
\hline LQSF(206).(207)RQDV & $2.1 \pm 0.5$ & $2.0 \pm 0.5$ & $3.5 \pm 0.2$ \\
\hline KSKF(295).(296)ADLS & $1.6 \pm 0.7$ & $2.0 \pm 0.6$ & $2.2 \pm 0.7$ \\
\hline SLTC(328).(329)EVDA & $2.8 \pm 1.0$ & $2.2 \pm 0.7$ & $4.7 \pm 0.5$ \\
\hline
\end{tabular}

B

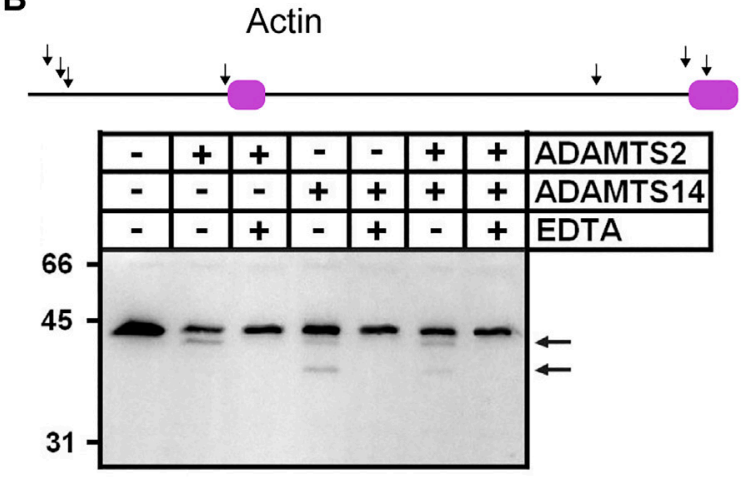

C

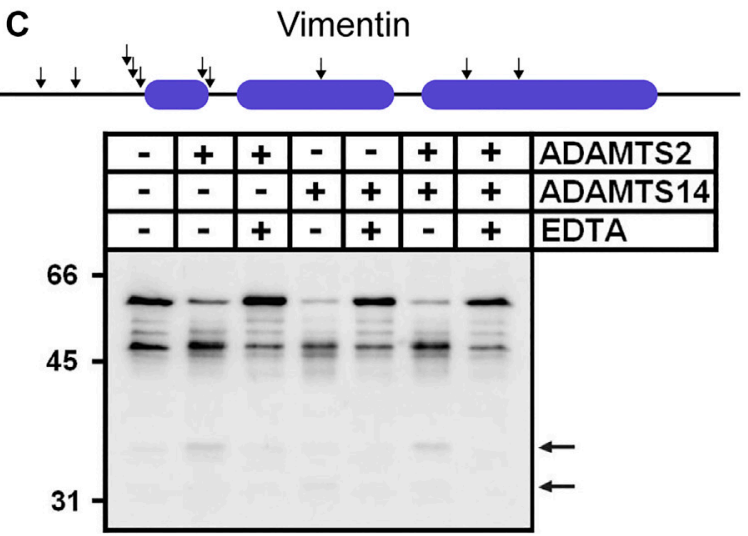

FIGURE 6 | In vitro cleavage of actin and vimentin by ADAMTS2 and ADAMTS14. Table showing the P/C ratios according to the cleavage sites observed by proteomic. Ratios above the average $3 \sigma$ cut-offs are highlighted in green (A). Western blot analysis of in vitro cleavage of actin (B) and vimentin (C) by ADAMTS2 and/or ADAMTS14. The schematic representation of actin and vimentin are reported on the top of the western blots with their respective actinin binding (magenta) and coiled coil (blue) domains. Human fibroblasts lysates were incubated in the presence of recombinant ADAMTS2 and/or ADAMTS14 (200 nM, $16 \mathrm{~h}$ at $37^{\circ} \mathrm{C}$ ). EDTA was used as an inhibitor of metalloproteinases. Degradation products are indicated by black arrows.

cleavage sites (Figure 7, upper panel). This revealed an overrepresentation of $\mathrm{G}$ and $\mathrm{P}$ that could be linked, at least in part, to the abundance of these amino acids in collagens. In addition, Y, F and A were often found at P1 and A and S at P1', which is similar to previously described cleavage sites for aminoprocollagen peptidases (Bekhouche et al., 2016; Janssen et al., 2016) (Figure 7, upper panel). Considering all the potential extracellular substrates, excluding type I collagen (middle panel) or all fibrillar collagens (lower panel), it was shown that ADAMTS2 and ADAMTS14 display common preferential cleavage sites, enriched in small nonpolar, amphipatic or slightly hydrophobic amino acids (G, P, F, Y, A, L and M). Of note also was the presence of acidic amino acids at P2', P3' and $\mathrm{P} 4$ '.

\section{DISCUSSION}

The procollagen $\mathrm{N}$-proteinases were originally described only for their ability to specifically excise the N-terminal propeptide of fibrillar collagens (Bekhouche and Colige, 2015). However, more recently, we and others have demonstrated their roles in TGF $\beta$ signaling (Bekhouche et al., 2016) and in lymphangiogenesis through the proteolytic activation of pro-VEGF-C into VEGF$\mathrm{C}$ able to interact with its receptors (Janssen et al., 2016; Brouillard et al., 2017; Dupont et al., 2018; Wang et al., 2020). The existence of marked differences between the phenotype of patients with arthrochalasia EDS (caused by the absence of the ADAMTS2 cleavage sites in type I collagen) and the phenotype of patients with dermatosparactic EDS (null mutations in ADAMTS2) is another observation arguing for additional functions of ADAMTS2. Finally, the peculiar atopic dermatitis-like skin phenotype of mice deficient in both ADAMTS2 and ADAMTS14 (Dupont et al., 2018) also points to yet to be discovered new substrates of these two related enzymes. In order to search for such potential substrates using a large-scale unbiased approach, we have compared $\mathrm{N}$-terminomes of the skin of Wild type, $\mathrm{TS}^{-/-}, \mathrm{TS}_{1} 4^{-/-}$and $\mathrm{TS} 2^{-/-} \mathrm{TS}_{14}{ }^{-/-}$mice, an experimental approach never tried before because its inherent difficulties, such as the dynamic nature of the cleavage events that could be hidden by cellular uptake, rapid degradation of the generated peptides, and potential variations in protein composition or cell abundance. Moreover, the deficiency of a protease in vivo can be compensated by another protease cleaving near or at the same cleavage site (Fortelny et al., 2014). As an additional challenge, skin is formed by several 


\section{ADAMTS2}
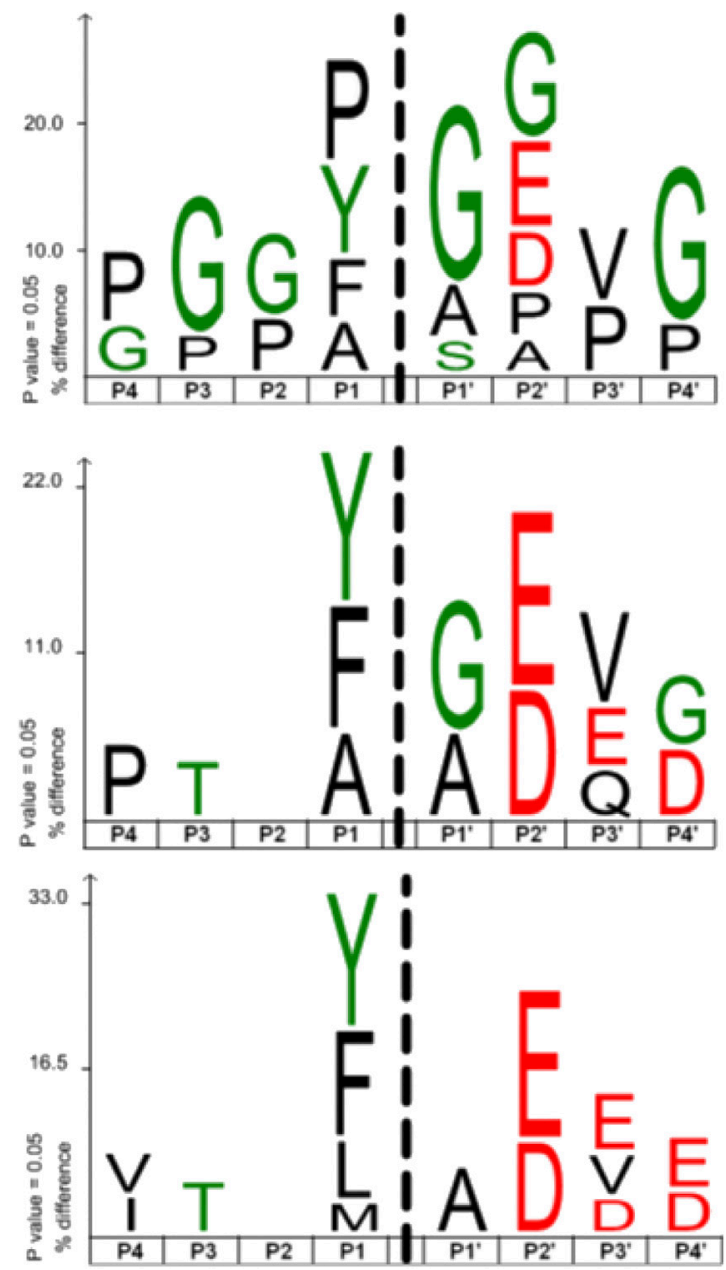

ADAMTS14
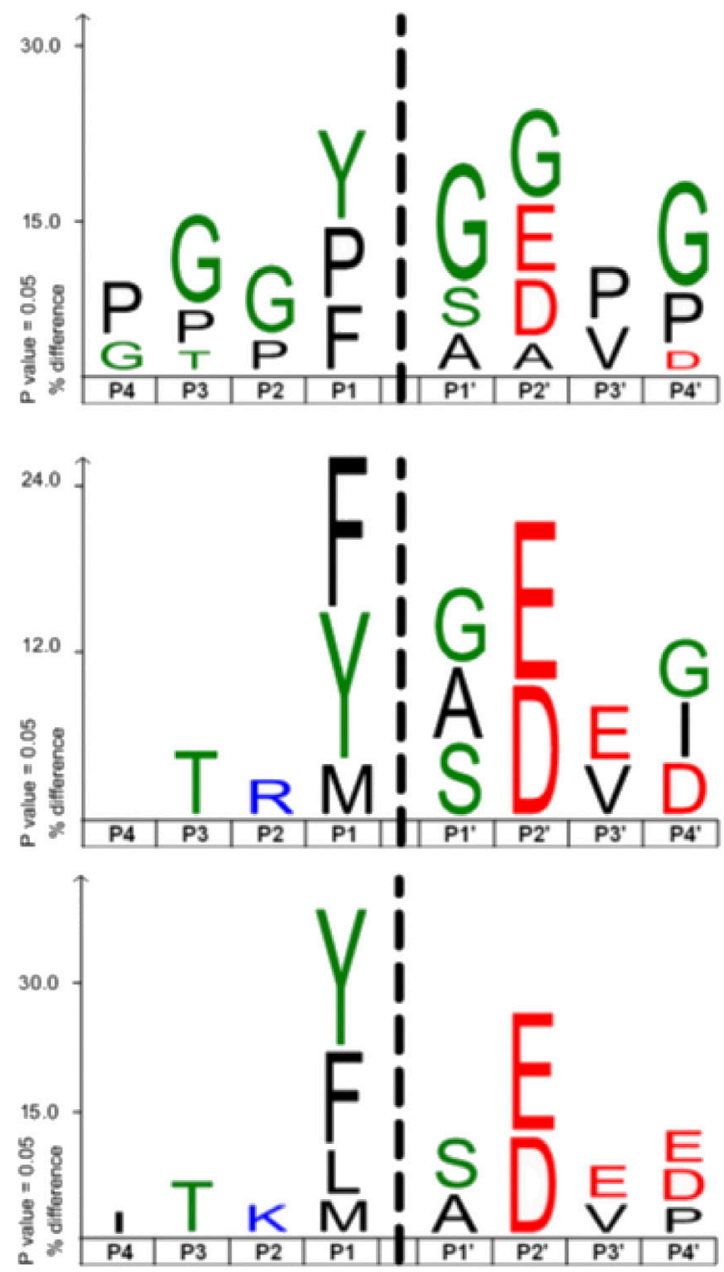

FIGURE 7 | Cleavage site specificity of ADAMTS2 and ADAMTS14 in mouse skin. The cleavage site specificity of ADAMTS2 (left panels) or ADAMTS14 (right panels) was determined using all the extracellular or extracellular transmembrane cleavage sites (top), without type I collagen (middle panels) or without all the fibrillar collagens (down panels). The amino acid sequence logos, corrected by the natural abundance of amino acids in the human proteome, were generated using the iceLogo software package (Colaert et al., 2009). The height of the amino acids represents their frequency regarding their natural abundance; the color reflects its physicochemical properties.

compartments such as epidermis, dermis, and muscular, nerve and blood vessels elements which may vary in abundance, a situation susceptible to induce modifications in the relative abundance of a specific protein. The use of a high throughput method and the complexity of the skin samples led to variations between the three individual experiments which prevented the identification of potential gender differences. In this study, three individual experiments have therefore been analyzed independently, in order to increase the likelihood of identifying new substrates, and have been compared between each other in a second step for validations purposes. Potential substrates were identified by taking into account only peptides harboring a $\mathrm{P} / \mathrm{C}$ ratio above or below the 3-sigma cutoff from the normal distribution of natural N-termini, which constitutes a very stringent threshold. Sixty-eight proteins located at the cell surface or being part of the extracellular matrix were identified as potential substrates of ADAMTS2 and/or ADAMTS14, from which fibrillary collagens were the most represented in terms of identified peptides.

The previously described cleavage sites by ADAMTS2 of the $\mathrm{N}$-propeptide of COL1A1 and COL1A2 were clearly identified, confirming the relevance of our experimental model. Of notes, however, these cleavages seem to occur within a preferential sequence rather than at a specific cleavage site as always reported so far, which illustrates that ADAMTS2 is capable to cleave multiple peptide bonds. As an additional evidence of the specificity of the N-TAILS data, cleavages of the N-propeptide of COL1A 1 and COL1A2 by ADAMTS14 were only marginal, which was expected from previous in vitro and in vivo data showing that ADAMTS14 displays only a very low aminoprocollagen peptidase activity (Colige et al., 2002; Dupont et al., 2018). Having clearly demonstrated the reliability of our experimental design, we then 
investigated the cleavages of the $\mathrm{N}$-propeptides of type $\mathrm{V}$ procollagens which are still incompletely defined. For COL5A1, a cleavage by BMP1 was previously identified within the NC3 domain (Imamura et al., 1998; Bonod-Bidaud et al., 2007) but could not be identified here as expected when using models comparing $\mathrm{Wt}$ to Adamts2 and/or Adamts14 deficient skins. The $\mathrm{P}_{435.436} \mathrm{~A}$ site previously found for ADAMTS2 at the end of the variable domain (Colige et al., 2005) was identified here for Adamts2 but also for Adamts14. Accordingly, the P/C ratio was much higher by comparing Wt skin to $\mathrm{TS}^{-1-} \mathrm{TS}^{-1} 4^{--}$skin, indicating that both enzymes display this activity in vivo and, therefore, identifying a new function for ADAMTS14. Some cleavages by both enzymes were also identified in the NC2 domain of COL5A1 and COL5A2 separating the small and the large central triple helical domains, at a location similar to the ones reported for type I, type II and type III procollagens, which was never described before. The existence of several cleavage sites generating type $\mathrm{V}$ collagens with $\mathrm{N}$-propeptides of different size and bulkiness is a new observation and is probably part of the regulation operated by type $\mathrm{V}$ collagen on collagen fibril formation (Linsenmayer et al., 1993; Marchant et al., 1996; Wenstrup et al., 2004) as observed in vitro and in vivo in EDS. It would also explain why arthrochalasic and dermatosparactic EDS have different clinical manifestations since both type I and type V collagen processing are altered in dermatosparaxis while only type I collagen is affected in arthrochalasia.

Besides cleavages of the N-propeptides, N-TAILS identified also several cleavages in the central COL1 domain of both alpha one and alpha 2 type I collagens. While some sites were not, or only barely, affected by the presence of ADAMTS2 or $14(\mathrm{P} / \mathrm{C}<1.5)$, some others were characterized by higher ratios, especially in some clusters, and when comparing Wt to $\mathrm{TS}^{-1-} \mathrm{TS}^{-14^{-/}}$skin, which suggested specificity in these cleavages. This hypothesis was evaluated in vitro using purified enzymes and purified collagen, either native or heat denatured. It showed that ADAMTS2 and ADAMTS14 can cleave within the COL1 triple helical domain, but mainly (or only) when collagen is unfolded. This "collagenase" activity was similar for ADAMTS2 and ADAMTS14, while ADAMTS14 displays only a limited capacity to cleave the aminopropeptide of non-denatured procollagen which demonstrates that the two activities are independent. The existence of such function was never described before and its biological relevance will have to be further investigated. An attractive hypothesis would be that, when ADAMTS2 (or ADAMTS14) meets and interacts with its type I procollagen substrate, it cleaves the $\mathrm{N}$-propeptide but it also degrades collagen trimers that are not correctly folded, therefore preventing their integration in fibrils. This quality control function of aminoprocollagen peptidases, if confirmed, would also explain why collagen fibrils have a so irregular and abnormal shape in dermatosparaxis. Indeed, in the absence of ADAMTS2, aminoprocollagen but also collagen with defects in the triple helix domains can both accumulate in fibrils and hamper the highly organized polymerization process of collagen fibrils.

Excision of the C-propeptide of type I procollagen was originally attributed to BMP1, and later extended to meprins $\alpha$ and $\beta$ (Kessler et al., 1996; Jefferson et al., 2013). Here, our degradomic analysis has revealed major cleavage sites for ADAMTS2 and ADAMTS14 in the C-propeptide of both COL1A1 and COL1A2. This was also confirmed in vitro using purified enzymes and type I procollagen, and is reminiscent to what was already demonstrated for type III procollagen (Bekhouche et al., 2016). However, the functional relevance of these observations in vivo, when BMP1 is present and active, is not clear yet since, except for one site in COL1A1, they are located downstream of the BMP1 site. In these conditions, these ADAMTS-dependent cleavages should not affect the maturation of type I collagen and its polymerization. Since the C-propeptide of type I collagen can inhibit collagen synthesis when released in the extracellular space (Mizuno et al., 2000), an intriguing hypothesis would be that its cleavage by ADAMTS2 or 14 would affect its regulatory function.

The main purpose of this study was to get a better insight into the overall implications of ADAMTS2 and ADAMTS14 in collagen fibril homeostasis. In addition of cleavages at multiple sites in fibrillary collagens, other proteins regulating collagen fibrillogenesis were also found to be potential substrates, similarly to what was found previously regarding the cleavage of Lox (Rosell-García et al., 2019), a crucial enzyme for the formation of crosslinks stabilizing collagen fibrils. Two cleavages were found in COL14A1, a FACIT (Fibril Associated Collagen with Interrupted Triple helix) known for its capacity to interact with collagen fibrils and regulate their formation (Young et al. 2002; Agarwal et al., 2012). Similarly, type VI collagen was also identified as a likely substrate regulating fibril formation (Lamandé and Bateman, 2018; Wu and Ge, 2019). However, the functional relevance of these cleavages was not investigated further but would deserve additional characterizations.

Lumican, biglycan and osteoglycin are members of the Small Leucin-rich Proteoglycan (SLRP) family (Frikeche et al., 2016). They are considered as collagen fibril-regulating proteins, and are also emerging as factors controlling immune response. It is interesting to note that these three proteoglycans have been identified here as potential substrates of ADAMTS2 which is also implicated in immune response as previously described (Hofer et al., 2008) and further illustrated here by the specific enrichment in biological processes linked to complement activation and regulation of cytokines involved in immune response. Although still to be considered as a working hypothesis and having to be confirmed by complementary approaches, these observations could pave the way to better understand the multiple roles of ADAMTS2 and 14 in extracellular matrix formation and functions.

Analysis of the skin degradome also identified cytoplasmic proteins, such as actin and vimentin, as potential substrates of ADAMTS2 and 14. These surprising data were confirmed in vitro using skin fibroblasts extracts incubated with recombinant purified enzymes, but not by comparing the electrophoretic pattern of actin and vimentin in Wt and ADAMTS2-deficient fibroblasts. This could suggest that these cleavages occur only after secretion or release in the extracellular space, as observed after cell death for example. We cannot however completely rule out the possibility that ADAMTS2 and 14 could display intracellular activities, either during the secretion process, as described for procollagen processing, or after their internalization when bound to cell membrane (Dubail et al., 
2010). The proteomic identification of intracellular substrates has already been reported for MMP2(auf dem Keller et al., 2013) and by studies reporting the intracellular functions of MMPs(Jobin et al., 2017), such as $\alpha$-actinin cleavage, or the transcriptional regulation of NF-kappa-B inhibitor alpha by MMP12(Marchant et al., 2014). Interestingly, these intracellular activities are related to the innate immunity system (Marchant et al., 2014) with a propensity for MMPs to regulate negatively the proinflammatory response (Dufour and Overall, 2013; Khokha et al., 2013). Further studies are needed to precisely assess the role the cleavage of proteins by ADAMTS2 and ADAMTS14 either in the extracellular and/or in the intracellular space, with a potential implication in cytoskeleton dynamic, gene expression and inflammatory response.

\section{CONCLUSION}

Our N-TAILS analysis of mouse skin degradomes has extended our knowledge regarding the roles of ADAMTS2 and 14 well beyond the previously known cleavage of the $\mathrm{N}$-propeptide of type I, type II and type III procollagens. The identification in vivo of several cleavage sites in the $\mathrm{N}$-terminal region of type $\mathrm{V}$ collagen, generating $\mathrm{N}$-terminal propeptides of different size and bulkiness, is a new finding possibly explaining how type $\mathrm{V}$ collagen can finely tune collagen fibril formation and structure. In the same context, other molecules involved in fibrillogenesis, such as SLRPs and non-fibrillar collagens, have also been identified as likely substrates of ADAMTS2 and 14.

Another intriguing observation is the potential quality control activity leading to the degradation of incorrectly folded collagen trimers before their assembly in collagen fibrils. If this hypothesis is confirmed, this would represent a key activity of ADAMTS2 and 14 for maintaining the structural integrity of collagen fibers and connective tissues.

\section{DATA AVAILABILITY STATEMENT}

The datasets presented in this study can be found in online repositories. The names of the repository/repositories and accession number(s) can be found in the article.

\section{REFERENCES}

Agarwal, P., Zwolanek, D., Keene, D. R., Schulz, J. N., Blumbach, K., Heinegård, D., et al. (2012). Collagen XII and XIV, new partners of cartilage oligomeric matrix protein in the skin extracellular matrix suprastructure. J. Biol. Chem. 287, 22549-22559. doi:10.1074/jbc.M111.335935

auf dem Keller, U., Prudova, A., Eckhard, U., Fingleton, B., and Overall, C. M. (2013). Systems-level analysis of proteolytic events in increased vascular permeability and complement activation in skin inflammation. Sci. Signal. 6, rs2. doi:10.1126/scisignal.2003512

auf dem Keller, U., and M Overall, C. (2012). CLIPPER: an add-on to the TransProteomic Pipeline for the automated analysis of TAILS N-terminomics data. Biol. Chem. 393, 1477-1483. doi:10.1515/hsz-2012-0269

\section{ETHICS STATEMENT}

The animal study was reviewed and approved by the Ethics Committee for Animal Use and Care, University of Liège, Belgium.

\section{AUTHOR CONTRIBUTIONS}

Conceptualization, $\mathrm{AC}$ and $\mathrm{MB}$; methodology, $\mathrm{MB}$; formal analysis, $\mathrm{AC} \mathrm{DB}$ and $\mathrm{MB}$; investigation, $\mathrm{CL} \mathrm{CM} \mathrm{LD} \mathrm{CD}$ and LJ; data curation, $\mathrm{DB}$ GM and $\mathrm{MB}$; writing original draft preparation, $\mathrm{MB}$ and $\mathrm{CL}$; writing review and editing, $\mathrm{AC}$ and $\mathrm{MB}$; visualization, $\mathrm{MB}$ and $\mathrm{AC}$; supervision, $\mathrm{MB}$ and $\mathrm{AC}$; project administration, $\mathrm{AC}$ and $\mathrm{MB}$; funding acquisition, $\mathrm{AC}$ and $\mathrm{MB}$. All authors have read and agreed to the published version of the manuscript.

\section{FUNDING}

The authors are grateful for support by the Incoming Postdoctoral-Marie Curie (COFUND) fellowship (Brussels, Belgium), the Télévie (7.4602.14; 7.4608.19; 7.4566.16), Fonds de la Recherche Scientifique-Fonds National de la Recherche Scientifique (FRS-FNRS; T.0183.13; CDR J003220 F).

\section{ACKNOWLEDGMENTS}

The authors thank Ulrich auf dem Keller (Technical University of Denmark, Denmark) for his help in running the analysis of $\mathrm{N}$-terminomics data, and Antoine Heyres for its technical support. We thank the Betty Nusgens for critical reading of the manuscript.

\section{SUPPLEMENTARY MATERIAL}

The Supplementary Material for this article can be found online at: https://www.frontiersin.org/articles/10.3389/fmolb.2021.643178/ full\#supplementary-material.

Beighton, P., De Paepe, A., Steinmann, B., Tsipouras, P., and Wenstrup, R. J. (1998). Ehlers-danlos syndromes: revised nosology, villefranche, 1997. Ehlersdanlos national foundation (United States) and ehlers-danlos support group (United Kindom). Am. J. Med. Genet. 77, 31-37. doi:10.1002/(sici)10968628(19980428)77:1<31::aid-ajmg8>3.0.co;2-o

Bekhouche, M., and Colige, A. (2015). The procollagen N-proteinases ADAMTS2, 3 and 14 in pathophysiology. Matrix Biol. 44-46, 46-53. doi:10.1016/j.matbio.2015.04.001

Bekhouche, M., Leduc, C., Dupont, L., Janssen, L., Delolme, F., Vadon-Le Goff, S., et al. (2016). Determination of the substrate repertoire of ADAMTS2, 3, and 14 significantly broadens their functions and identifies extracellular matrix organization and TGF- $\beta$ signaling as primary targets. FASEB J. 30, 1741-1756. doi:10.1096/fj.15-279869

Bonod-Bidaud, C., Beraud, M., Vaganay, E., Delacoux, F., Font, B., Hulmes, D. J., et al. (2007). Enzymatic cleavage specificity of the proalphal(V) chain 
processing analysed by site-directed mutagenesis. Biochem. J. 405, 299-306. doi:10.1042/BJ20070051

Bradford, M. M. (1976). A rapid and sensitive method for the quantitation of microgram quantities of protein utilizing the principle of protein-dye binding. Anal. Biochem. 72, 248-254. doi:10.1006/abio.1976.9999

Brouillard, P., Dupont, L., Helaers, R., Coulie, R., Tiller, G. E., Peeden, J., et al. (2017). Loss of ADAMTS3 activity causes Hennekam lymphangiectasialymphedema syndrome 3. Hum. Mol. Genet. 26, 4095-4104. doi:10.1093/ $\mathrm{hmg} / \mathrm{ddx} 297$

Colaert, N., Helsens, K., Martens, L., Vandekerckhove, J., and Gevaert, K. (2009). Improved visualization of protein consensus sequences by iceLogo. Nat. Methods 6, 786-787. doi:10.1038/nmeth1109-786

Colige, A., Beschin, A., Samyn, B., Goebels, Y., Van Beeumen, J., Nusgens, B. V., et al. (1995). Characterization and partial amino acid sequencing of a $107-\mathrm{kDa}$ procollagen I N-proteinase purified by affinity chromatography on immobilized type XIV collagen. J. Biol. Chem. 270, 16724-16730. doi:10. $1074 / j b c .270 .28 .16724$

Colige, A., Sieron, A. L., Li, S. W., Schwarze, U., Petty, E., Wertelecki, W., et al. (1999). Human Ehlers-Danlos syndrome type VII C and bovine dermatosparaxis are caused by mutations in the procollagen I N-proteinase gene. Am. J. Hum. Genet. 65, 308-317. doi:10.1086/302504

Colige, A., Vandenberghe, I., Thiry, M., Lambert, C. A., Van Beeumen, J., Li, S. W., et al. (2002). Cloning and characterization of ADAMTS-14, a novel ADAMTS displaying high homology with ADAMTS-2 and ADAMTS-3. J. Biol. Chem. 277, 5756-5766. doi:10.1074/jbc.M105601200

Colige, A., Nuytinck, L., Hausser, I., Van Essen, A. J., Thiry, M., Herens, C., et al. (2004). Novel types of mutation responsible for the dermatosparactic type of Ehlers-Danlos syndrome (Type VIIC) and common polymorphisms in the ADAMTS2 gene. J. Invest. Dermatol. 123, 656-663. doi:10.1111/j.0022-202X.2004.23406.x

Colige, A., Ruggiero, F., Vandenberghe, L., Dubail, J., Kesteloot, F., Van Beeumen, J., et al. (2005). Domains and maturation processes that regulate the activity of ADAMTS-2, a metalloproteinase cleaving the aminopropeptide of fibrillar procollagens types I-III and V. J. Biol. Chem. 280, 34397-34408. doi:10. 1074/jbc.M506458200

De Paepe, A., and Malfait, F. (2012). The Ehlers-Danlos syndrome, a disorder with many faces. Clin. Genet. 82, 1-11. doi:10.1111/j.1399-0004.2012.01858.x

Dubail, J., Kesteloot, F., Deroanne, C., Motte, P., Lambert, V., Rakic, J. M., et al. (2010). ADAMTS-2 functions as anti-angiogenic and anti-tumoral molecule independently of its catalytic activity. Cell. Mol. Life Sci. 67, 4213-4232. doi:10. 1007/s00018-010-0431-6

Dufour, A., and Overall, C. M. (2013). Missing the target: matrix metalloproteinase antitargets in inflammation and cancer. Trends Pharmacol. Sci. 34, 233-242. doi:10.1016/j.tips.2013.02.004

Dupont, L., Ehx, G., Chantry, M., Monseur, C., Leduc, C., Janssen, L., et al. (2018). Spontaneous atopic dermatitis due to immune dysregulation in mice lacking Adamts2 and 14. Matrix Biol. 70, 140-157. doi:10.1016/j.matbio.2018.04.002

Fortelny, N., Cox, J. H., Kappelhoff, R., Starr, A. E., Lange, P. F., Pavlidis, P., et al. (2014). Network analyses reveal pervasive functional regulation between proteases in the human protease web. PLoS Biol. 12, e1001869. doi:10.1371/ journal.pbio. 1001869

Frikeche, J., Maiti, G., and Chakravarti, S. (2016). Small leucine-rich repeat proteoglycans in corneal inflammation and wound healing. Exp. Eye Res. 151, 142-149. doi:10.1016/j.exer.2016.08.015

Hofer, T. P. J., Frankenberger, M., Mages, J., Lang, R., Meyer, P., Hoffmann, R., et al. (2008). Tissue-specific induction of ADAMTS2 in monocytes and macrophages by glucocorticoids. J. Mol. Med. 86, 323-332. doi:10.1007/ s00109-007-0284-0

Hulsen, T., de Vlieg, J., and Alkema, W. (2008). BioVenn-a web application for the comparison and visualization of biological lists using area-proportional Venn diagrams. BMC Genomics 9, 488. doi:10.1186/1471-2164-9-488

Imamura, Y., Steiglitz, B. M., and Greenspan, D. S. (1998). Bone morphogenetic protein-1 processes the $\mathrm{NH}$-terminal propeptide, and a furin-like proprotein convertase processes the $\mathrm{COOH}$-terminal propeptide of pro-alphal(V) collagen. J. Biol. Chem. 273, 27511-27517. doi:10.1074/jbc.273.42.27511

Janssen, L., Dupont, L., Bekhouche, M., Noel, A., Leduc, C., Voz, M., et al. (2016). ADAMTS3 activity is mandatory for embryonic lymphangiogenesis and regulates placental angiogenesis. Angiogenesis 19, 53-65. doi:10.1007/s10456015-9488-Z
Jefferson, T., Auf dem Keller, U., Bellac, C., Metz, V. V., Broder, C., Hedrich, J., et al. (2013). The substrate degradome of meprin metalloproteases reveals an unexpected proteolytic link between meprin $\beta$ and ADAM10. Cell. Mol. Life Sci. 70, 309-333. doi:10.1007/s00018-012-1106-2

Jobin, P. G., Butler, G. S., and Overall, C. M. (2017). New intracellular activities of matrix metalloproteinases shine in the moonlight. Biochim. Biophys. Acta Mol. Cell Res. 1864, 2043-2055. doi:10.1016/j.bbamcr.2017.05.013

Kessler, E., Takahara, K., Biniaminov, L., Brusel, M., and Greenspan, D. S. (1996). Bone morphogenetic protein-1: the type I procollagen C-proteinase. Science 271, 360-362. doi:10.1126/science.271.5247.360

Kesteloot, F., Desmoulière, A., Leclercq, I., Thiry, M., Arrese, J. E., Prockop, D. J., et al. (2007). ADAM metallopeptidase with thrombospondin type 1 motif 2 inactivation reduces the extent and stability of carbon tetrachloride-induced hepatic fibrosis in mice. Hepatology 46, 1620-1631. doi:10.1002/hep.21868

Khokha, R., Murthy, A., and Weiss, A. (2013). Metalloproteinases and their natural inhibitors in inflammation and immunity. Nat. Rev. Immunol. 13, 649-665. doi:10.1038/nri3499

Kleifeld, O., Doucet, A., Prudova, A., auf dem Keller, U., Gioia, M., Kizhakkedathu, J. N., et al. (2011). Identifying and quantifying proteolytic events and the natural $\mathrm{N}$ terminome by terminal amine isotopic labeling of substrates. Nat. Protoc. 6, 1578-1611. doi:10.1038/nprot.2011.382

Lamandé, S. R., and Bateman, J. F. (2018)Collagen VI disorders: insights on form and function in the extracellular matrix and beyond. Matrix Biol. 71-72, 348-367. doi:10.1016/j.matbio.2017.12.008

Linsenmayer, T. F., Gibney, E., Igoe, F., Gordon, M. K., Fitch, J. M., Fessler, L. I., et al. (1993). Type $V$ collagen: molecular structure and fibrillar organization of the chicken alpha $1(\mathrm{~V}) \mathrm{NH} 2$-terminal domain, a putative regulator of corneal fibrillogenesis. J. Cell Biol. 121, 1181-1189. doi:10.1083/jcb.121.5.1181

Malfait, F., and De Paepe, A. (2014). The Ehlers-Danlos syndrome. Adv. Exp. Med. Biol. 802, 129-143. doi:10.1007/978-94-007-7893-1_9

Marchant, J. K., Hahn, R. A., Linsenmayer, T. F., and Birk, D. E. (1996). Reduction of type $\mathrm{V}$ collagen using a dominant-negative strategy alters the regulation of fibrillogenesis and results in the loss of corneal-specific fibril morphology. J. Cell Biol. 135, 1415-1426. doi:10.1083/jcb.135.5.1415

Marchant, D. J., Bellac, C. L., Moraes, T. J., Wadsworth, S. J., Dufour, A., Butler, G. S., et al. (2014). A new transcriptional role for matrix metalloproteinase-12 in antiviral immunity. Nat. Med. 20, 493-502. doi:10.1038/nm.3508

Mi, H., Muruganujan, A., Ebert, D., Huang, X., and Thomas, P. D. (2019). PANTHER version 14: more genomes, a new PANTHER GO-slim and improvements in enrichment analysis tools. Nucleic Acids Res. 47, D419-D426. doi:10.1093/nar/gky1038

Mizuno, M., Fujisawa, R., and Kuboki, Y. (2000). The effect of carboxyl-terminal propeptide of type I collagen (c-propeptide) on collagen synthesis of preosteoblasts and osteoblasts. Calcif. Tissue Int. 67, 391-399. doi:10.1007/s002230001150

Nusgens, B., and Lapiere, C. M. (1979). A simplified procedure for measuring amino-procollagen peptidase type I. Anal. Biochem. 95, 406-412. doi:10.1016/ 0003-2697(79)90747-4

Nusgens, B. V., Verellen-Dumoulin, C., Hermanns-Lê, T., De Paepe, A., Nuytinck, L., Piérard, G. E., et al. (1992). Evidence for a relationship between EhlersDanlos type VII C in humans and bovine dermatosparaxis. Nat. Genet. 1, 214-217. doi:10.1038/ng0692-214

Perez-Riverol, Y., Csordas, A., Bai, J., Bernal-Llinares, M., Hewapathirana, S., Kundu, D. J., et al. (2019). The PRIDE database and related tools and resources in 2019: improving support for quantification data. Nucleic Acids Res. 47. D442-D450. doi:10.1093/nar/gky1106

Poeter, M., Brandherm, I., Rossaint, J., Rosso, G., Shahin, V., Skryabin, B. V., et al. (2014). Annexin A8 controls leukocyte recruitment to activated endothelial cells via cell surface delivery of CD63. Nat. Commun. 5, 3738. doi:10.1038/ ncomms 4738

Romano, E., Rosa, I., Fioretto, B. S., Lucattelli, E., Innocenti, M., Ibba-Manneschi, L., et al. (2020). A two-step immunomagnetic microbead-based method for the isolation of human primary skin telocytes/cd34+ stromal cells. Int. J. Mol. Sci. 21, 5877. doi:10.3390/ijms21165877

Rosell-García, T., Paradela, A., Bravo, G., Dupont, L., Bekhouche, M., Colige, A., et al. (2019). Differential cleavage of lysyl oxidase by the metalloproteinases BMP1 and ADAMTS2/14 regulates collagen binding through a tyrosine sulfate domain. J. Biol. Chem. 294, 11087-11100. doi:10.1074/jbc.ra119. 007806 
Sugimoto, M. A., Vago, J. P., Teixeira, M. M., and Sousa, L. P. (2016). Annexin A1 and the resolution of inflammation: modulation of neutrophil recruitment, apoptosis, and clearance. J. Immunol. Res. 2016, 8239258. doi:10.1155/2016/ 8239258

Swisher, J. F., Khatri, U., and Feldman, G. M. (2007). Annexin A2 is a soluble mediator of macrophage activation. J. Leukoc. Biol. 82, 1174-1184. doi:10.1189/ jlb.0307154

Van Damme, T., Colige, A., Syx, D., Giunta, C., Lindert, U., Rohrbach, M., et al. (2016). Expanding the clinical and mutational spectrum of the Ehlers-Danlos syndrome, dermatosparaxis type. Genet. Med. 18, 882-891. doi:10.1038/gim. 2015.188

Wang, G., Muhl, L., Padberg, Y., Dupont, L., Peterson-Maduro, J., Stehling, M., et al. (2020). Specific fibroblast subpopulations and neuronal structures provide local sources of Vegfc-processing components during zebrafish lymphangiogenesis. Nat. Commun. 11, 2724. doi:10.1038/s41467-020-16552-7

Wenstrup, R. J., Florer, J. B., Brunskill, E. W., Bell, S. M., Chervoneva, I., and Birk, D. E. (2004). Type V collagen controls the initiation of collagen fibril assembly. J. Biol. Chem. 279, 53331-53337. doi:10.1074/jbc.M409622200
Wu, Y., and Ge, G. (2019). Complexity of type IV collagens: from network assembly to function. Biol. Chem. 400, 565-574. doi:10.1515/hsz-20180317

Young, B. B., Zhang, G., Koch, M., and Birk, D. E. (2002). The roles of types XII and XIV collagen in fibrillogenesis and matrix assembly in the developing cornea. J. Cell Biochem. 87, 208-220. doi:10.1002/jcb.10290

Conflict of Interest: The authors declare that the research was conducted in the absence of any commercial or financial relationships that could be construed as a potential conflict of interest.

Copyright (c) 2021 Leduc, Dupont, Joannes, Monseur, Baiwir, Mazzucchelli, Deroanne, Colige and Bekhouche. This is an open-access article distributed under the terms of the Creative Commons Attribution License (CC BY). The use, distribution or reproduction in other forums is permitted, provided the original author(s) and the copyright owner(s) are credited and that the original publication in this journal is cited, in accordance with accepted academic practice. No use, distribution or reproduction is permitted which does not comply with these terms. 